\title{
SELECTIVE STRONG SCREENABILITY
}

\author{
by \\ Isaac Joseph Coombs
}

\author{
A thesis \\ submitted in partial fulfillment \\ of the requirements for the degree of \\ Master of Science in Mathematics \\ Boise State University
}

August 2018 
(C) 2018

Isaac Joseph Coombs

ALL RIGHTS RESERVED 
BOISE STATE UNIVERSITY GRADUATE COLLEGE

DEFENSE COMMITTEE AND FINAL READING APPROVALS

of the thesis submitted by

Isaac Joseph Coombs

Thesis Title: Selective Strong Screenability

Date of Final Oral Examination: 03 May 2012

The following individuals read and discussed the thesis submitted by student Isaac Joseph Coombs, and they evaluated his presentation and response to questions during the final oral examination. They found that the student passed the final oral examination.

Marion Scheepers, Ph.D. Chair, Supervisory Committee

Liljana Babinkostova, Ph.D. Member, Supervisory Committee

Andrés Caicedo, Ph.D. Member, Supervisory Committee

The final reading approval of the thesis was granted by Marion Scheepers, Ph.D., Chair, Supervisory Committee. The thesis was approved for the Graduate College. 


\begin{abstract}
Screenability and strong screenability were both introduced some sixty years ago by R.H. Bing in his paper Metrization of Topological Spaces. Since then, much work has been done in exploring selective screenability (the selective version of screenability). However, the corresponding selective version of strong screenability has been virtually ignored. In this paper we seek to remedy this oversight. It is found that a great deal of the proofs about selective screenability readily carry over to proofs for the analogous version for selective strong screenability. We give some examples of selective strongly screenable spaces with the primary example being Pol's space. We go on to explore a natural weakening of selective strong screenability in topological groups. We conclude with an exploration of the difficulty in extending discrete families of sets as well as giving several directions one might go in when continuing the exploration of selective strong screenability.
\end{abstract}




\section{TABLE OF CONTENTS}

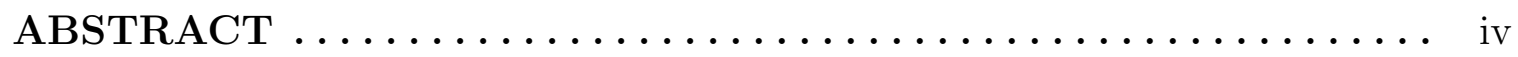

1 Introduction $\ldots \ldots \ldots \ldots \ldots \ldots \ldots \ldots \ldots \ldots \ldots \ldots \ldots \ldots \ldots \ldots \ldots \ldots$

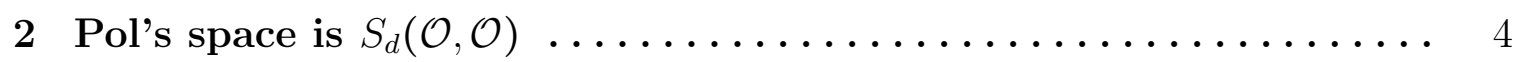

2.1 Every finite power of the reals is an $S_{d}(\mathcal{O}, \mathcal{O})$-space $\ldots \ldots \ldots \ldots \ldots$

2.2 Compact, finite dimensional metric spaces are $S_{d}(\mathcal{O}, \mathcal{O}) \ldots \ldots \ldots \ldots$

2.3 The countable sum of compact $S_{d}(\mathcal{O}, \mathcal{O})$-spaces $\ldots \ldots \ldots \ldots \ldots .17$

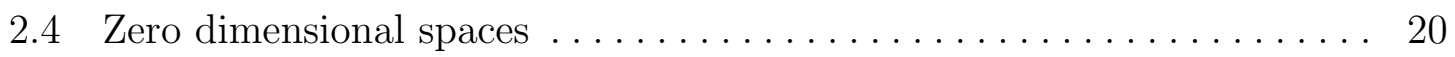

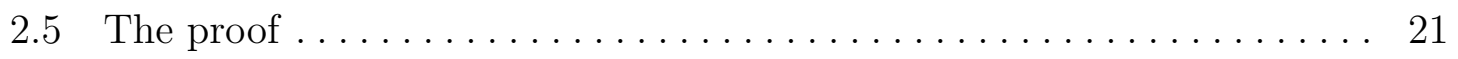

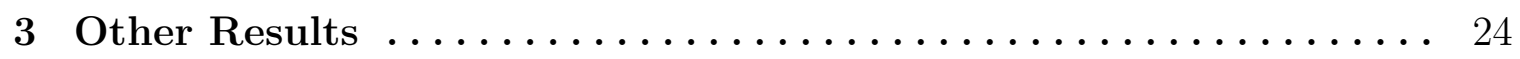

3.1 Generalizations of earlier results $\ldots \ldots \ldots \ldots \ldots \ldots \ldots \ldots \ldots \ldots \ldots \ldots$

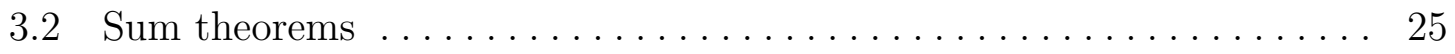

3.3 More product theorems ............................ 27

3.4 Another relation between strong Haver and $S_{d}(\mathcal{O}, \mathcal{O}) \ldots \ldots \ldots \ldots$

3.5 Topological Groups ............................... 32

3.6 Relation to other selection principles ................... 43

\section{Open Questions}

and where we go from here $\ldots \ldots \ldots \ldots \ldots \ldots \ldots \ldots \ldots \ldots \ldots$

4.1 The Discrete Extension Question ....................... 46 


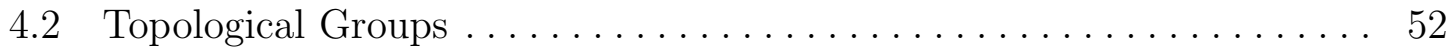

4.3 Relation to other Selection principles $\ldots \ldots \ldots \ldots \ldots \ldots \ldots$

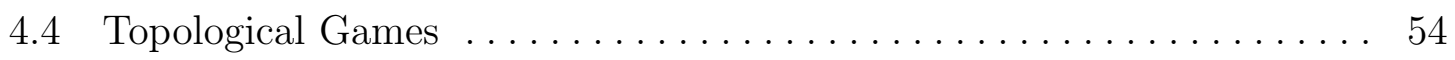

REFERENCES ........................ 56 


\section{CHAPTER 1}

\section{INTRODUCTION}

In 1951 in [9], R.H. Bing explored when a topological space is metrizable. In his paper, he introduced several important types of spaces. Among these we find screenable and strongly screenable spaces. A space is screenable if for each open cover $\mathcal{U}$ of the space there is a sequence $\left(\mathcal{V}_{n}: n<\infty\right)$ such that for each $n, \mathcal{V}_{n}$ is a pairwise disjoint family

of open sets refining $\mathcal{U}$ and $\bigcup_{n<\infty} \mathcal{V}_{n}$ is a cover of the space. Note that for families $\mathcal{A}$ and $\mathcal{B}$, we will use the notation $\mathcal{A}<\mathcal{B}$, read " $\mathcal{A}$ refines $\mathcal{B}$ ", to denote that for each $A \in \mathcal{A}$ there is a $B \in \mathcal{B}$ such that $A \subseteq B$. Recall that a family $\mathcal{F}$ of subsets of a topological space $X$ is said to be a discrete family if each point in $X$ has a neighborhood which has nontrivial intersection with at most one element of $\mathcal{F}$. Bing called a space strongly screenable if instead of each $\mathcal{V}_{n}$ being a pairwise disjoint family of open sets, each $\mathcal{V}_{n}$ is a discrete family of open sets. Further, Bing showed that for metrizable spaces, screenability is equivalent to strong screenability. It s natural to ask if the same is true for the selective versions, introduced next.

In 1973 in [16], Haver introduced a covering property of metric spaces which he called property $C$, but which we refer to as the Haver property. In 1978 in [1], Addis and Gresham extended Haver's property $C$ to one for general topological spaces. A topological space, say $X$, is said to have property $C$ if for each sequence $\left(\mathcal{U}_{n}: n<\infty\right)$ of open covers of $X$ there is a sequence $\left(\mathcal{V}_{n}: n<\infty\right)$ such that for each $n, \mathcal{V}_{n}$ is a 
pairwise disjoint family of open sets which refines $\mathcal{U}_{n}$ and $\bigcup_{n<\infty} \mathcal{V}_{n}$ is a cover of $X$. In 2001 in [2], Liljana Babinkostova introduced the selection principle $S_{c}(\mathcal{A}, \mathcal{B})$, where $\mathcal{A}$ and $\mathcal{B}$ are subsets of $\mathcal{P}(X)$. A topological space $X$ is said to have property $S_{c}(\mathcal{A}, \mathcal{B})$ if for each sequence $\left(\mathcal{U}_{n}: n<\infty\right)$ of elements of $\mathcal{A}$, there is a sequence $\left(\mathcal{V}_{n}: n<\infty\right)$ such that each $\mathcal{V}_{n}$ refines $\mathcal{U}_{n}$, each $\mathcal{V}_{n}$ is a pairwise disjoint family of sets, and $\bigcup_{n<\infty} \mathcal{V}_{n}$ is an element of $\mathcal{B}$. When $\mathcal{A}=\mathcal{B}=\mathcal{O}$, where $\mathcal{O}$ denotes the collection of all open covers of $X$, then $S_{c}(\mathcal{O}, \mathcal{O})$, read "ess see oh oh", is what Babinkostova called selective screenability. Selective screenability is the same as Addis and Gresham's property $C$, and so we now refer to their version of $C$-spaces as $S_{c}(\mathcal{O}, \mathcal{O})$-spaces.

There is a great deal known about spaces that are selectively screenable. For instance, see [1], [10], [11], [13], [14], [15], [?], [22], and [23]. However, there is much less known about selective strong screenablity.

Definition 1: A topological space $X$ is said to be selectively strongly screenable if for each sequence $\left(\mathcal{U}_{n}: n<\infty\right)$ of open covers of $X$ there exists a corresponding sequence $\left(\mathcal{V}_{n}: n<\infty\right)$ such that

(i) For each $n, \mathcal{V}_{n}$ is a discrete family of sets open in $X$,

(ii) For each $n, \mathcal{V}_{n}<\mathcal{U}_{n}$, and

(iii) $\bigcup_{n<\infty} \mathcal{V}_{n}$ is a cover of $X$

We will often use $S_{d}(\mathcal{O}, \mathcal{O})$-space in place of "selectively strongly screenable space", and other similar abbreviations. We will also summarize conditions (i), (ii) and (iii) by saying that the sequence $\left(\mathcal{V}_{n}: n<\infty\right)$ is an $S_{d}(\mathcal{O}, \mathcal{O})$-refinement $\left(\right.$ of $\left.\left(\mathcal{U}_{n}: n<\infty\right)\right)$. With this in mind, we say that a topological space is $S_{d}(\mathcal{O}, \mathcal{O})$ if each sequence of open covers of the space admits an $S_{d}(\mathcal{O}, \mathcal{O})$-refinement. 
For this thesis, we set out to expand what is known about $S_{d}(\mathcal{O}, \mathcal{O})$-spaces. To this end, we examined some of the known results about $S_{c}(\mathcal{O}, \mathcal{O})$-spaces and whether their proofs would still hold if one replaced all occurrences of "pairwise disjoint family" with "discrete family" (and other similar substitutions as necessary). In the majority of the cases examined, we found that the proofs given for selective screenability would, in essence, extend directly to a proof of the corresponding result for selective strong screenability. Proofs that are not direct extensions of those given in the literature for the selective screenable case will be indicated using $a \star$. For those that did extend directly, we will include in a footnote for each proof providing both the author and paper from which our extended proof came. Additionally, we will give some discussion of what part of the proofs needed additional results, or where we had difficulties (and failed in at least one important case) in proving the $S_{d}(\mathcal{O}, \mathcal{O})$ analog of a known $S_{c}(\mathcal{O}, \mathcal{O})$ result. The culmination of this exploration came in showing that Pol's space, a space of importance in dimension theory, is selectively strongly screenable. In the next chapter we will describe Pol's space in more detail and work our way towards proving that it is selectively strongly screenable. 


\section{CHAPTER 2}

\section{POL'S SPACE IS $S_{d}(\mathcal{O}, \mathcal{O})$}

In 1981 in [25], R. Pol gave an example of a weakly infinite-dimensional compact space that is not countable-dimensional. This space has come to be known as Pol's space. It builds on results of Mazurkiewicz and Lelek. In 1927, Mazurkiewicz constructed a complete, totally disconnected, strongly infinite dimensional metric space, which we will denote by $M$. In 1965, Lelek gave the existence of the compactification of a complete metric space by a space, which we will denote by $L$, and is the countable union of a family of compact, finite dimensional spaces. The result, $M \cup L$, is Pol's space, denoted by $P$. In this chapter, we give the necessary theorems used in the proof that Pol's space is $S_{d}(\mathcal{O}, \mathcal{O})$, according to the following outline.

Let $\left(\mathcal{U}_{n}: n<\infty\right)$ be a sequence of open covers of $P$. We will show that every finite power of the reals is $S_{d}(\mathcal{O}, \mathcal{O})$, that each closed subspace of an $S_{d}(\mathcal{O}, \mathcal{O})$-space is also an $S_{d}(\mathcal{O}, \mathcal{O})$-space, and then use the Menger-Nöbeling Theorem (see page 420 of [19]) to conclude that every compact finite dimensional metric space is an $S_{d}(\mathcal{O}, \mathcal{O})$-space. We then use a technique developed in showing that the countable union of compact $S_{d}(\mathcal{O}, \mathcal{O})$-spaces is an $S_{d}(\mathcal{O}, \mathcal{O})$-space to find an $S_{d}(\mathcal{O}, \mathcal{O})$-refinement of $\left(\mathcal{U}_{n}: n>1\right)$, say $\left(\mathcal{V}_{n}: n>1\right)$, whose union covers $L$. We then see that $P \backslash \bigcup\left(\bigcup_{n>1} \mathcal{V}_{n}\right)$ is a closed, and hence compact, subspace of $P$, and more specifically of $M$. We then use that a compact, totally disconnected metric space is zero-dimensional (Theorem 29.7 of [29]) 
to see that $P \backslash \bigcup\left(\bigcup_{n>1} \mathcal{V}_{n}\right)$ is zero dimensional. Then we use the zero dimensionality of $P \backslash \bigcup\left(\bigcup_{n>1} \mathcal{V}_{n}\right)$ to find an $S_{d}(\mathcal{O}, \mathcal{O})$-refinement, $\mathcal{V}_{1}$ of $\mathcal{U}_{1}$. Taken together, $\left(\mathcal{V}_{n}: n<\infty\right)$ is an $S_{d}(\mathcal{O}, \mathcal{O})$-refinement of $\left(\mathcal{U}_{n}: n<\infty\right)$ witnessing that Pol's space is selectively strongly screenable.

\subsection{Every finite power of the reals is an $S_{d}(\mathcal{O}, \mathcal{O})$-space}

The Hurewicz and Haver properties are well known and, as illustrated by Babinkostova in [4], they can be used to determine if a space is $S_{c}(\mathcal{O}, \mathcal{O})$. A topological space $X$ has the Hurewicz property if for each sequence $\left(\mathcal{U}_{n}: n<\infty\right)$ of open covers of $X$ there is a corresponding sequence $\left(\mathcal{V}_{n}: n<\infty\right)$ such that for each $n, \mathcal{V}_{n}$ is a finite subset of $\mathcal{U}_{n}$ and each element of $X$ is in almost every $\bigcup \mathcal{V}_{n}$. Throughout, we use the term "almost every" to mean "in all but finitely many". A metric space $(X, d)$ is said to have the Haver property if for each sequence $\left(\varepsilon_{n}: n<\infty\right)$ of positive real numbers there is a corresponding sequence $\left(\mathcal{V}_{n}: n<\infty\right)$ such that: for each $n, \mathcal{V}_{n}$ is a pairwise disjoint family of sets open in $X$; for each $n$, for each $V \in \mathcal{V}_{n}$, the $d$-diameter of $V$ is less than $\varepsilon_{n}$; and, $\bigcup_{n<\infty} \mathcal{V}_{n}$ is a cover of $X$. The theorems presented in this section are, in essence, merely the corresponding strong version of many of the product theorems (and lemmas needed for them) in [4].

For $S_{d}(\mathcal{O}, \mathcal{O})$, selective strong screenability, recall that we are merely working with the strong version of $S_{c}(\mathcal{O}, \mathcal{O})$, selective screenability, i.e. we consider discrete families instead of pairwise disjoint families in all corresponding definitions. Thus, instead of the Haver property, we will look at the strong Haver property. A metric space $(X, d)$ is said to have the strong Haver property if for each sequence $\left(\varepsilon_{n}: n<\infty\right)$ of positive real numbers there is a corresponding sequence $\left(\mathcal{V}_{n}: n<\infty\right)$ such that: 
for each $n, \mathcal{V}_{n}$ is a discrete family of sets open in $X$; for each $n$, for each $V \in \mathcal{V}_{n}$, the $d$-diameter of $V$ is less than $\varepsilon_{n}$; and, $\bigcup_{n<\infty} \mathcal{V}_{n}$ is a cover of $X$.

These properties, the Hurewicz and strong Haver property, are sufficient conditions for a metric space to be an $S_{d}(\mathcal{O}, \mathcal{O})$-space.

Theorem 1: If $(X, d)$ is a metric space with both the Hurewicz property and the strong Haver property, then it is an $S_{d}(\mathcal{O}, \mathcal{O})$-space.

In $\S 3.4$, Theorem 14 , we will see that a metric space $(X, d)$ is selectively strongly screenable if, and only if, it has the strong Haver property in each equivalent metric. Thus, we see that for a metric space $(X, d)$, the strong Haver property with respect to the metric $d$ is a necessary condition for the metric space to be an $S_{d}(\mathcal{O}, \mathcal{O})$-space. With Theorem 14 in mind, we then see that

Corollary 1: For a metric space $(X, d)$ with the strong Haver property with respect to the metric $d$, if $X$ also has the Hurewicz property, then $X$ has the strong Haver property in all equivalent metrics.

Proof of Theorem 1: ${ }^{1}$ Let $\left(\mathcal{U}_{n}: n<\infty\right)$ be a sequence of open covers of $X$ and define

$$
\mathcal{H}_{n}:=\left\{B(x, \epsilon): \epsilon>0, x \in X \text {, and }\left(\exists U \in \mathcal{U}_{n}\right)(B(x, 2 \epsilon) \subseteq U)\right\}
$$

Using the Hurewicz property on $X$, for each $n$ we choose a finite set $\mathcal{F}_{n} \subseteq \mathcal{H}_{n}$ such that each element of $X$ is in almost every $\cup \mathcal{F}_{n}$. We will represent each set as $\mathcal{F}_{n}=$ $\left\{B\left(x_{i}^{n}, \epsilon_{i}^{n}\right): i \in I_{n}\right\}$, where $I_{n}$ is a finite indexing set for $\mathcal{F}_{n}$. Define

\footnotetext{
${ }^{1}$ This proof follows that given by Babinkostova of Theorem 5 in [4]. The key property of pairwise disjoint families used in Babinkostova's proof is that a subset of a pairwise disjoint family is also pairwise disjoint. Since discrete families also have the property that a subset of a discrete family is also discrete, there was really little to do for this result.
} 


$$
\varepsilon_{n}:=\min \left\{\epsilon_{i}^{n}: i \in I_{n}\right\}
$$

Now, partition the natural numbers into infinitely many sets, each of which is infinite, say $\mathbb{N}=\bigcup_{k<\infty} J_{k}$. Apply the strong Haver property of $X$ for the metric $d$ on $X$ to each of the sequences $\left(\varepsilon_{n}: n \in J_{k}\right)$ to find for each $k$ a sequence $\left(\mathcal{S}_{n}: n \in J_{k}\right)$ such that each $\mathcal{S}_{n}$ is a discrete family of open subsets of $X$, each of diameter less than $\varepsilon_{n}$, and $\bigcup_{n \in J_{k}} \mathcal{S}_{n}$ is a cover of $X$. For each $n$ define

$$
\mathcal{V}_{n}=\left\{S \in \mathcal{S}_{n}:\left(\exists U \in \mathcal{U}_{n}\right)(S \subseteq U)\right\}
$$

Since each $\mathcal{S}_{n}$ is a discrete family of sets open in $X$ and $\mathcal{V}_{n} \subseteq \mathcal{S}_{n}$, we have that each $\mathcal{V}_{n}$ is a discrete family of sets open in $X$ and refining $\mathcal{U}_{n}$.

It remains to show that $\bigcup_{n<\infty} \mathcal{V}_{n}$ is a cover of $X$. Let $x \in X$ be arbitrary. Since each element of $X$ is in almost every $\cup \mathcal{F}_{n}$, we pick an $N_{x} \in \mathbb{N}$ large enough that for each $n \geq N_{x}$ we have that $x \in \cup \mathcal{F}_{n}$. By our choice of partitioning the natural numbers, we pick a $k \in \mathbb{N}$ such that $N_{x}<\min J_{k}$. Since $\bigcup_{n \in J_{k}} \mathcal{S}_{n}$ is a cover of $X$, we pick an $m \in J_{k}$ such that $x \in \cup S_{m}$. Now, we pick $S \in \mathcal{S}_{m}$ such that $x \in S$. Notice that since $N_{x}<\min J_{k}$ and $m \in J_{k}$ we have that $m>N_{x}$, and thus $x \in \cup \mathcal{F}_{m}$. Next, we choose $i \in I_{m}$ such that $x \in B\left(x_{i}^{m}, \varepsilon_{i}^{m}\right) \in \mathcal{F}_{m}$. Since $\mathcal{F}_{m} \subseteq \mathcal{H}_{m}$ we pick $U \in \mathcal{U}_{m}$ such that $B\left(x_{i}^{m}, 2 \varepsilon_{i}^{m}\right) \subseteq U$. Consider any $y \in S$ and notice that

$$
d\left(y, x_{i}^{m}\right) \leq d(x, y)+d\left(x, x_{i}^{m}\right) \leq \operatorname{diam} S+\varepsilon_{i}^{m}<\varepsilon_{m}+\varepsilon_{i}^{m} \leq \varepsilon_{i}^{m}+\varepsilon_{i}^{m}=2 \varepsilon_{i}^{m} .
$$

Thus, we see that $y \in B\left(x_{i}^{m}, 2 \varepsilon_{i}^{m}\right) \subseteq U$. Therefore $S \subseteq U$ and so $S \in \mathcal{V}_{m}$, thereby showing that $\bigcup_{n<\infty} \mathcal{V}_{n}$ is a cover of $X$. 
Notice that a key to the above proof was the ability to partition the natural numbers into infinitely many infinite sets. This technique is used frequently.

It is well known that

Fact 1: Each $\sigma$-compact space has the Hurewicz property.

This was proven by Hurewicz when he introduced this property in his 1927 paper, [17]. It is also well known that

Fact 2: The set of reals with the standard topology is $\sigma$-compact, and each finite power of a $\sigma$-compact space is $\sigma$-compact.

Taken together, this gives us that

Fact 3: Each finite power of the reals has the Hurewicz property.

It is straightforward to see that

Lemma 1: The reals have the strong Haver property.

Proof. Let $\left(\varepsilon_{n}: n<\infty\right)$ be a sequence of positive reals. Pick positive real numbers $\varepsilon<\varepsilon_{1}$ and $\delta<\min \left\{\varepsilon, \varepsilon_{2}\right\}$. Then define

$$
\mathcal{V}_{n}:= \begin{cases}\left\{B\left(k\left(\varepsilon+\frac{\delta}{2}\right), \varepsilon\right): k \in \mathbb{Z}\right\} & \text { if } n=1 \\ \left\{B\left(\frac{\varepsilon}{2}+\frac{\delta}{4}+k\left(\varepsilon+\frac{\delta}{2}\right), \delta\right): k \in \mathbb{Z}\right\} & \text { if } n=2 \\ \varnothing & \text { if } n \geq 3 .\end{cases}
$$

Notice that each $\mathcal{V}_{n}$ is a discrete family of sets open in the reals and that each element of $\mathcal{V}_{n}$ has diameter less than $\varepsilon_{n}$. Hence $\left(\mathcal{V}_{n}: n<\infty\right)$ witnesses that $\mathbb{R}$ has the strong Haver property. 
For an example of the above construction of $\mathcal{V}_{n}$, suppose that $\varepsilon_{1}=1$ and $\varepsilon_{2}=1$. Then, Figure 2.1 illustrates $\mathcal{V}_{1}$ and $\mathcal{V}_{2}$, where we take $\varepsilon=\frac{3}{4}$ and $\delta=\frac{1}{2}$

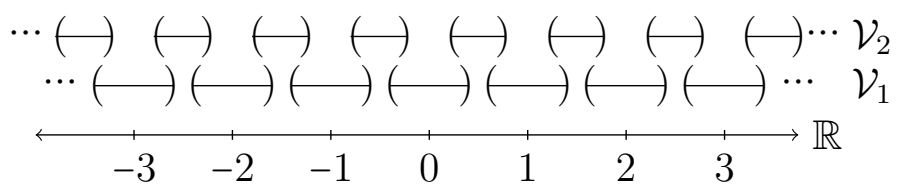

Figure 2.1: Illustration for Lemma 1.

Hence, by Fact 3 and Lemma 1 applied to Theorem 1 we see that

Lemma 2: The set of reals with the standard topology is an $S_{d}(\mathcal{O}, \mathcal{O})$-space.

To see that all finite powers of the reals are $S_{d}(\mathcal{O}, \mathcal{O})$, we will need the following theorem about when the product of two strong Haver spaces is again a strong Haver space, which is analogous to Theorem 8 of [4].

Theorem 2: Let $X$ and $Y$ be metric spaces with the strong Haver property. If either $X$ or $Y$ has the $\sigma$-discrete grouping property, then $X \times Y$ has the strong Haver property.

In [4] Babinkostova introduced the $\sigma$-disjoint grouping property for ease of handling the Haver property. A topological space has the $\sigma$-disjoint grouping property if for each sequence $\left(\mathcal{U}_{n}: n<\infty\right)$ such that each $\mathcal{U}_{n}$ is a pairwise disjoint family of open sets and for each $x \in X$ there are infinitely many $n$ with $x \in \bigcup \mathcal{U}_{n}$, there is a corresponding strictly increasing sequence $\left(n_{k}: k<\infty\right)$ such that for each $x \in X$, for all but finitely many $k, x \in \bigcup\left(\bigcup_{n_{k} \leq i<n_{k+1}} \mathcal{U}_{i}\right)$. For handling the strong Haver property, we will use the $\sigma$-discrete grouping property, which is the corresponding strong version of $\sigma$-disjoint grouping property. A topological space has the $\sigma$-discrete grouping property if for each sequence $\left(\mathcal{U}_{n}: n<\infty\right)$ such that each $\mathcal{U}_{n}$ is a discrete family of open sets 
and for each $x \in X$ there are infinitely many $n$ with $x \in \bigcup \mathcal{U}_{n}$, there is a corresponding strictly increasing sequence $\left(n_{k}: k<\infty\right)$ such that for each $x \in X$, for all but finitely many $k, x \in \bigcup\left(\bigcup_{n_{k} \leq i<n_{k+1}} \mathcal{U}_{i}\right)$.

Before proving Theorem 2, we need to show that the cover given by the strong Haver property may be taken to be a large cover. Recall that a cover $\mathcal{U}$ of a space $X$ is said to be a large cover if each element of $X$ is contained in infinitely many elements of $\mathcal{U}$. Further, recall for a topological space $X$ that an element $x \in X$ is an isolated point if there is an open neighborhood $N_{x}$ of $x$ such that $x$ is the only element of $N_{x}$. In particular, notice that an isolated point has a neighborhood with zero diameter.

Lemma 3: For a metric space $(X, d)$ with no isolated points the following are equivalent:

(1) $(X, d)$ has the strong Haver property.

(2) For each sequence $\left(\varepsilon_{n}: n<\infty\right)$ of positive real numbers there is a sequence $\left(\mathcal{U}_{n}: n<\infty\right)$ such that each $\mathcal{U}_{n}$ is a discrete family of open sets, each of diameter less than $\varepsilon_{n}$, and $\bigcup_{n<\infty} \mathcal{U}_{n}$ is a large cover of $X$.

Proof. ${ }^{2}$ To see that $(1) \Rightarrow(2)$ do the following: Let $\left(\varepsilon_{n}: n<\infty\right)$ be a sequence of positive real numbers. It may be assumed that this sequence is strictly decreasing and that it converges to zero. To see this, notice that if $\left(\varepsilon_{n}: n<\infty\right)$ is not decreasing and convergent to zero, then we may define

$$
\delta_{n}= \begin{cases}\varepsilon_{n} & n=0 \\ \frac{1}{2}\left(\min \left(\left\{\varepsilon_{i}: i \leq n\right\} \cup\left\{\delta_{i}: i<n\right\}\right)\right) & n>0 .\end{cases}
$$

\footnotetext{
${ }^{2}$ This proof follows that given of Lemma 7 in [4].
} 
Apply the strong Haver property to the sequence $\left(\delta_{n}: n<\infty\right)$, which is strictly decreasing, to obtain a sequence $\left(\mathcal{U}_{n}: n<\infty\right)$ of discrete families of open sets such that for each $n$, the diameter of each element of $\mathcal{U}_{n}$ is less than $\delta_{n}$ and $\bigcup_{n<\infty} \mathcal{U}_{n}$ is a cover of the space. Since $\delta_{n} \leq \varepsilon_{n}$, this sequence of discrete families also works for $\left(\varepsilon_{n}: n<\infty\right)$

Let $\bigcup_{k<\infty} I_{k}$ be a partition of the natural numbers into infinitely many infinite sets. Apply the strong Haver property of $(X, d)$ to each of the sequences $\left(\delta_{n}: n \in I_{k}\right)$ to obtain a sequence $\left(\mathcal{U}_{n}: n \in I_{k}\right)$ of discrete families of open sets such that for each $n$, the diameter of each element of $\mathcal{U}_{n}$ is less than $\delta_{n}$ and $\bigcup_{n \in I_{k}} \mathcal{U}_{n}$ is a cover of $X$. It remains to show that $\bigcup_{n<\infty} \mathcal{U}_{n}$ is a large cover of $X$.

Fix any $y \in X$ and $n \in \mathbb{N}$. Assume we have already selected sets $U_{1}, \ldots, U_{n}$ such that for each $j \leq n$ there is an $m_{j}$ and $U_{j} \in \bigcup_{i \in I_{m_{j}}} \mathcal{U}_{i}$ with $y \in U_{j}$. That we can find a first such set is clear since $\bigcup_{n<\infty} \mathcal{U}_{n}$ is a cover of $X$. To find $U_{n+1} \notin\left\{U_{1}, \ldots U_{n}\right\}$ with $y \in U_{n+1}$ and $U_{n+1} \in \bigcup_{n<\infty} \mathcal{U}_{n}$, do as follows.

First, set $\delta=\min \left\{\operatorname{diam}\left(U_{j}\right): j \leq n\right\}$. Choose $k \in \mathbb{N}$ such that $\delta_{k}<\delta$; this can be done since $\left(\delta_{n}: n<\infty\right)$ converges to zero and since $\delta>0$ (which follows from $X$ having no isolated points). Next, by our partitioning of the natural numbers, choose $\ell$ such that $\min \left(I_{\ell}\right)>k$. Since $\bigcup_{i \in I_{\ell}} \mathcal{U}_{i}$ covers $X$, we choose $U_{n+1}$ to be a member of this cover which contains $y$. By our choice of $\ell$ we have that $\operatorname{diam}\left(U_{n+1}\right)<\delta_{k}<\delta$. Therefore, we have that $U_{n+1} \notin\left\{U_{1}, \ldots, U_{n}\right\}$. Thus, we see that $\bigcup_{n<\infty} \mathcal{U}_{n}$ has infinitely many distinct members containing $y$, and so conclude that $\bigcup_{n<\infty} \mathcal{U}_{n}$ is a large cover of $X$.

That $(2) \Rightarrow(1)$ is clear since every large cover of $X$ is certainly a cover of $X$.

In many of the proofs given about products of spaces for the $S_{c}(\mathcal{O}, \mathcal{O})$ case, it 
was used that the product of two pairwise disjoint families is also a pairwise disjoint family. The corresponding version which allows the $S_{c}(\mathcal{O}, \mathcal{O})$ proofs to work for the strong case is

Lemma $4(\star)$ : For discrete families $\mathcal{A}$ and $\mathcal{B}$ of subsets of topological spaces $X$ and $Y$, respectively, the family $\mathcal{C}=\{A \times B: A \in \mathcal{A}$ and $B \in \mathcal{B}\}$ is a discrete family of subsets of $X \times Y$.

Proof. Let $\mathcal{A}, \mathcal{B}$, and $\mathcal{C}$ be as given. Consider any $(x, y) \in X \times Y$. Since $\mathcal{A}$ is a discrete family of sets in $X$ there is a neighborhood $N_{x}$ of $x$ such that at most one element of $\mathcal{A}$ has non-trivial intersection with $N_{x}$. Since $\mathcal{B}$ is a discrete family of sets in $Y$ there is a neighborhood $N_{y}$ of $y$ such that at most one element of $\mathcal{B}$ has non-trivial intersection with it. Notice that $N_{x} \times N_{y}$ is a neighborhood of $(x, y)$ in $X \times Y$. Suppose there were distinct elements $A \times B$ and $A^{\prime} \times B^{\prime}$ of $\mathcal{C}$ such that their intersection with $N_{x} \times N_{y}$ were non-trivial. If $A \times B$ and $A^{\prime} \times B^{\prime}$ are distinct because $A \neq A^{\prime}$, then $A$ and $A^{\prime}$ are distinct members of $\mathcal{A}$ which have non-trivial intersection with $N_{x}$. This, however, contradicts our choice of $N_{x}$, which we found since $\mathcal{A}$ is a discrete family. Hence we see that it must be that $A=A^{\prime}$. Similarly, we see that $B=B^{\prime}$, and so $A \times B=A^{\prime} \times B^{\prime}$, contradicting our assumption that they were distinct members of $\mathcal{C}$ with nontrivial intersection with $N_{x} \times N_{y}$, i.e. that $\mathcal{C}$ was not a discrete family. Hence, we may conclude that $\mathcal{C}$ is a discrete family of sets in $X \times Y$.

We are now ready to prove Theorem 2 .

Proof of Theorem 2: ${ }^{3}$ Let $\left(\varepsilon_{n}: n<\infty\right)$ be a strictly decreasing sequence of positive real numbers. Since $Y$ has the strong Haver property, by applying Lemma 3 to the sequence $\left(\frac{\varepsilon_{n}}{2}: n<\infty\right)$, we obtain a sequence $\left(\mathcal{U}_{n}: n<\infty\right)$ such that each $\mathcal{U}_{n}$ is a

\footnotetext{
${ }^{3}$ This proof follows that given by Babinkostova of Theorem 8 of [4].
} 
discrete family of sets open in $Y$, each of diameter less than $\frac{\varepsilon_{n}}{2}$, and $\bigcup_{n<\infty} \mathcal{U}_{n}$ is a large cover of $Y$. Since each $\mathcal{U}_{n}$ is a discrete family of open sets, each $y \in Y$ is found in at most a single element of $\mathcal{U}_{n}$. Hence, since $\bigcup_{n<\infty} \mathcal{U}_{n}$ is a large cover of $Y$ we see that each $y \in Y$ is in $\bigcup \mathcal{U}_{n}$ for infinitely many $n$. By the $\sigma$-discrete grouping property of $Y$ applied to $\left(\mathcal{U}_{n}: n<\infty\right)$ we obtain a strictly increasing sequence $\left(n_{k}: k<\infty\right)$ of natural numbers such that each $y \in Y$ is in almost every $\bigcup\left(\bigcup_{n_{k} \leq j<n_{k+1}} \mathcal{U}_{j}\right)$.

Now, for each $k$ define $\delta_{k}=\frac{\varepsilon_{n_{k}}}{2}$. Since $X$ has the strong Haver property, by applying Lemma 3 to the sequence $\left(\delta_{k}: k<\infty\right)$, we obtain a sequence $\left(\mathcal{V}_{k}: k<\infty\right)$ such that each $\mathcal{V}_{k}$ is a discrete family of sets open in $X$, each of diameter less than $\delta_{k}$, and $\bigcup_{k<\infty} \mathcal{V}_{k}$ is a large cover of $X$.

Define the following families of sets:

$$
\mathcal{W}_{i}= \begin{cases}\left\{V \times U: V \in \mathcal{V}_{1} \text { and } U \in \mathcal{U}_{i}\right\}, & i<n_{1} \\ \left\{V \times U: V \in \mathcal{V}_{k+1} \text { and } U \in \mathcal{U}_{i}\right\}, & n_{k} \leq i<n_{k+1}\end{cases}
$$

By Lemma 4, we know that each $\mathcal{W}_{i}$ is a discrete family of sets open in $X \times Y$. By the standard product metric and use of the triangle inequality we see that for $W \in \mathcal{W}_{i}$ we have for $i<n_{1}$ that

$$
\begin{aligned}
\operatorname{diam} W & =\sqrt{(\operatorname{diam} V)^{2}+(\operatorname{diam} U)^{2}}<\sqrt{\left(\delta_{1}\right)^{2}+\left(\frac{\varepsilon_{i}}{2}\right)^{2}} \\
& =\sqrt{\left(\frac{\varepsilon_{n_{1}}}{2}\right)^{2}+\left(\frac{\varepsilon_{i}}{2}\right)^{2}}<\sqrt{\left(\frac{\varepsilon_{i}}{2}\right)^{2}+\left(\frac{\varepsilon_{i}}{2}\right)^{2}} \\
& =\frac{\sqrt{2}}{2} \varepsilon_{i}
\end{aligned}
$$

and for $n_{k} \leq i<n_{k+1}$ that 


$$
\begin{aligned}
\operatorname{diam} W & =\sqrt{(\operatorname{diam} V)^{2}+(\operatorname{diam} U)^{2}}<\sqrt{\left(\delta_{k+1}\right)^{2}+\left(\frac{\varepsilon_{i}}{2}\right)^{2}} \\
& =\sqrt{\left(\frac{\varepsilon_{n_{k+1}}}{2}\right)^{2}+\left(\frac{\varepsilon_{i}}{2}\right)^{2}}<\sqrt{\left(\frac{\varepsilon_{i}}{2}\right)^{2}+\left(\frac{\varepsilon_{i}}{2}\right)^{2}} \\
& =\frac{\sqrt{2}}{2} \varepsilon_{i} .
\end{aligned}
$$

In either case the elements of $\mathcal{W}_{i}$ are of diameter less that $\varepsilon_{i}$. It remains to show that $\bigcup_{i<\infty} \mathcal{W}_{i}$ is a cover of $X \times Y$.

Let $(x, y) \in X \times Y$. Since each $y \in Y$ is in almost every $\bigcup\left(\bigcup_{n_{k} \leq j<n_{k+1}} \mathcal{U}_{j}\right)$, we pick an $N$ large enough that for each $k \geq N$ we have $y \in \bigcup\left(\bigcup_{n_{k} \leq j<n_{k+1}} \mathcal{U}_{j}\right)$. Since each $\mathcal{V}_{k}$ is a discrete family of sets and since $\bigcup_{k<\infty} \mathcal{V}_{k}$ is a large cover, we choose a $k>N$ with $x \in \bigcup \mathcal{V}_{k+1}$. Thus, we have $(x, y) \in V \times U \in \mathcal{V}_{k+1} \times \mathcal{U}_{i} \in \mathcal{W}_{i}$ for some $i$ with $n_{k} \leq i<n_{k+1}$. Hence, $\bigcup_{i<\infty} \mathcal{W}_{i}$ is a cover of $X \times Y$. Therefore, we conclude that $X \times Y$ has the strong Haver property.

To see that all finite powers of the reals have the $\sigma$-discrete grouping property, we turn to the following facts and lemma. In [28] Scheepers showed that

Fact 4: If a topological space has the Hurewicz property, then it has the grouping property.

A space $X$ is said to have the grouping property if for each bijectively enumerated large cover $\left(U_{n}: n<\infty\right)$ there is a sequence $n_{1}<n_{2}<\cdots<n_{k}<\cdots$ such that each element of $X$ is in all but finitely many of the sets $\bigcup_{n_{k} \leq j<n_{k+1}} U_{j}$. In [4] Babinkostova showed that

Fact 5: The grouping property is equivalent to the $\sigma$-disjoint grouping property.

Since each discrete family is a disjoint family, it is evident that 
Lemma 5: The $\sigma$-disjoint grouping property implies the $\sigma$-discrete grouping property.

With these facts and lemma in mind, we see that a space with the Hurewicz property also has the $\sigma$-discrete grouping property. In particular, every finite power of the reals has the $\sigma$-discrete grouping property. Hence, as a corollary to Theorem 2, we see that all finite powers of the reals have the strong Haver property. Thus we see that

Theorem 3: Each finite power of the set of reals with the standard topology is an $S_{d}(\mathcal{O}, \mathcal{O})$-space.

Hence, as more general corollaries to Theorem 2 we have

Corollary 2: If $X$ and $Y$ have the strong Haver property and either $X$ or $Y$ has the Hurewicz property, then $X \times Y$ has the strong Haver property.

Simple induction arguments then give:

Corollary 3: If $X$ is a strong Haver space with the $\sigma$-discrete grouping property, then all finite powers of $X$ have the strong Haver property.

Corollary 4: If $X$ has both the strong Haver and Hurewicz properties, then all finite powers of $X$ have the strong Haver property.

\subsection{Compact, finite dimensional metric spaces are $S_{d}(\mathcal{O}, \mathcal{O})$}

Our next objective on the way to showing that Pol's space is $S_{d}(\mathcal{O}, \mathcal{O})$ is to show that every compact, finite dimensional metric space is an $S_{d}(\mathcal{O}, \mathcal{O})$-space. The keys to this are the Menger-Nöbeling Theorem and the following theorem. 
Theorem 4 (Closed Subspace Theorem, *): Every closed subspace of an $S_{d}(\mathcal{O}, \mathcal{O})$ space is also an $S_{d}(\mathcal{O}, \mathcal{O})$-space.

Proof. Let $X$ be an $S_{d}(\mathcal{O}, \mathcal{O})$-space and let $C \subseteq X$ be closed. Let $\left(\mathcal{U}_{n}: n<\infty\right)$ be a sequence of open (in the relative topology on $C$ ) covers of $C$. For each $n$ and each $U \in \mathcal{U}_{n}$ fix an open set $U^{\prime} \subseteq X$ such that $U=C \cap U^{\prime}$ and define

$$
W_{U}:=(X \backslash C) \cup U^{\prime}
$$

Notice that since $C$ is closed in $X, X \backslash C$ is open in $X$, and hence $W_{U}$ is open in $X$. Next, define the family

$$
\mathcal{W}_{n}:=\left\{W_{U}: U \in \mathcal{U}_{n}\right\}
$$

Since for each $U \in \mathcal{U}_{n}$ we have $X \backslash C \subseteq W_{U}$ and $U \subseteq W_{U}$, we see that each $\mathcal{W}_{n}$ is an open cover of $X$. Applying $S_{d}(\mathcal{O}, \mathcal{O})$ of $X$ to the sequence $\left(\mathcal{W}_{n}: n<\infty\right)$ of open covers of $X$, we obtain $\left(\mathcal{V}_{n}: n<\infty\right)$, an $S_{d}(\mathcal{O}, \mathcal{O})$-refinement of $\left(\mathcal{W}_{n}: n<\infty\right)$. Now, for each $n$ define

$$
\mathcal{V}_{n}^{\prime}:=\left\{V \cap C: V \in \mathcal{V}_{n}\right\}
$$

It is clear that for each $n, \mathcal{V}_{n}^{\prime}$ is a discrete family of sets open in the relative topology on $C$ and refining $\mathcal{U}_{n}$. Further, since $\bigcup_{n<\infty} \mathcal{V}_{n}$ is a cover of $X$, we have that $\bigcup_{n<\infty} \mathcal{V}_{n}^{\prime}$ is a cover of $C$. Thus, $\left(\mathcal{V}_{n}^{\prime}: n<\infty\right)$ is an $S_{d}(\mathcal{O}, \mathcal{O})$-refinement of $\left(\mathcal{U}_{n}: n<\infty\right)$, and so $C$ is an $S_{d}(\mathcal{O}, \mathcal{O})$-space.

Having the Closed Subspace Theorem, we may now use

Theorem 5 (Menger-Nöbeling Theorem, pg 420 of [19]): Every separable metric space of dimension $n$ is homeomorphic to a subspace of $\mathbb{R}^{2 n+1}$. 
Recalling that compact metric spaces are separable we have by the MengerNöbeling Theorem that if $\mathrm{C}$ is a compact $n$-dimensional metric space, then $C$ is homeomorphic to a subspace, say $C^{\prime}$, of $\mathbb{R}^{2 n+1}$. In particular, $C^{\prime}$ is compact, and hence a closed subspace of $\mathbb{R}^{2 n+1}$. By Theorem $3, \mathbb{R}^{2 n+1}$ is an $S_{d}(\mathcal{O}, \mathcal{O})$-space. By the Closed Subspace Theorem, $C^{\prime}$ is an $S_{d}(\mathcal{O}, \mathcal{O})$-space. Thus, since it is clear that $S_{d}(\mathcal{O}, \mathcal{O})$ is preserved by homeomorphisms, $C$ is an $S_{d}(\mathcal{O}, \mathcal{O})$-space. Hence, we have Theorem 6: Every compact, finite dimensional metric space is an $S_{d}(\mathcal{O}, \mathcal{O})$-space.

\subsection{The countable sum of compact $S_{d}(\mathcal{O}, \mathcal{O})$-spaces}

From [1], we know that

If $X$ is a metric space such that $X=\bigcup_{n<\infty} X_{n}$ and each $X_{n}$ is an $S_{c}(\mathcal{O}, \mathcal{O})$ space (in the relative topology), then $X$ is an $S_{c}(\mathcal{O}, \mathcal{O})$-space.

In the proof given by Addis and Gresham, they use the idea that if $X \subseteq Y$ and $\mathcal{F}$ is a disjoint family of sets open in $X$, then $\mathcal{F}$ extends "nicely" to a disjoint family of sets open in $Y$. More explicitly, they use what amounts to Theorem II.21.XI.2 of [19], which states

Let $Y$ be a metric space and $X \subseteq Y$. Given a family $\left\{U_{\iota}\right\}$ of sets open in $X$, there exists a family $\left\{V_{\iota}\right\}$ of open (in $Y$ ) sets such that $X \cap V_{\iota}=U_{\iota}$ and the condition $U_{\iota_{1}} \cap \cdots \cap U_{\iota_{n}}=\varnothing$ implies $V_{\iota_{1}} \cap \cdots \cap V_{\iota_{n}}=\varnothing$, for every (finite) system of indices $\iota_{1}, \ldots, \iota_{n}$.

Unfortunately, we have not yet found a way to nicely extend discrete families in the subspace topology to discrete families in the superspace topology. We will discuss this in more detail in Chapter 4. Thus far, the closest we have come to an analogous version of the countable union of $S_{c}(\mathcal{O}, \mathcal{O})$-spaces theorem is 
Theorem $7(\star)$ : If $X=\bigcup_{n<\infty} X_{n}$ where each $X_{n}$ is a compact $S_{d}(\mathcal{O}, \mathcal{O})$-space, then $X$ is an $S_{d}(\mathcal{O}, \mathcal{O})$-space.

While this theorem will not be directly applicable in proving that Pol's space is selectively strongly screenable, the technique of its proof, and the tools discovered on the way to proving it are very much applicable in proving that result. The key to this theorem is the special case that each finite discrete family of sets in the relative topology extends nicely to a discrete family of sets in the superspace topology. Note that by "superspace" we mean that if $X$ is a subspace of a topological space $Y$, then we say that $Y$ is a superspace of $X$. Recall that a topological space $X$ is said to be normal if for each pair of disjoint closed sets $C_{1}, C_{2} \subseteq X$, there are disjoint open sets $U_{1}$ and $U_{2}$ such that $C_{1} \subseteq U_{1}$ and $C_{2} \subseteq U_{2}$.

Lemma $6(\star)$ : Let $Y$ be a normal topological space and let $X \subseteq Y$ be closed. For $\mathcal{F}=\left\{F_{1}, \ldots, F_{n}\right\} \subseteq \mathcal{P}(X)$, a finite discrete family of sets open in the relative topology on $X$, there exists $\mathcal{F}^{\prime}=\left\{U_{1}, \ldots U_{n}\right\} \subseteq \mathcal{P}(Y)$, a discrete family of sets open in $Y$ such that $F_{i} \subseteq U_{i}$ for each $i$.

Proof. Let $X, Y$, and $\mathcal{F}$ be as given. We proceed by induction on $n$, the number of elements in $\mathcal{F}$. If $\mathcal{F}$ is either empty or contains only a single set, then we can take $\mathcal{F}^{\prime}=\{Y\}$ and $\mathcal{F}^{\prime}$ is as desired. So, suppose that $n \geq 2$. We will construct $\mathcal{F}^{\prime}=\left\{U_{1}, \ldots U_{n}\right\}$ recursively as follows. Since $\mathcal{F}$ is a discrete family of sets open in $X$, we know that $\left\{\overline{F_{i}^{X}}: 1 \leq i \leq n\right\}$ is a pairwise disjoint family of sets closed in $X$. Since $X$ is a closed subset of $Y$, we also know that each $\overline{F_{i}^{X}}=\overline{F_{i}^{Y}}$ and so is closed in $Y$. We will now construct open (in $Y$ ) sets $U_{1}$ and $U_{2}$ about $F_{1}$ and $F_{2}$, respectively, such that $\overline{U_{1}^{Y}} \cap \overline{U_{2}^{Y}}=\varnothing$. 
We have that $\overline{F_{1}^{Y}} \cap \overline{F_{2}^{Y}}=\varnothing$. Since $Y$ is a normal topological space, we find disjoint open (in $Y$ ) sets $V_{1} \supset \overline{F_{1}^{Y}}$ and $V_{2} \supset \overline{F_{2}^{Y}}$ such that $V_{1} \cap V_{2}=\varnothing$. Since $V_{1} \cap V_{2}=\varnothing$, we know that $\overline{V_{1}^{Y}} \cap \overline{V_{2}^{Y}} \subseteq \partial V_{1}^{Y} \cap \partial V_{2}^{Y} \cdot{ }^{4}$ Since $\overline{F_{2}^{Y}} \subset V_{2}$ and $V_{2} \cap \partial V_{2}^{Y}=\varnothing$, we know that $\overline{V_{1}^{Y}} \cap \overline{F_{2}^{Y}}=\varnothing$ Again, using normality of $Y$, we find disjoint open (in $Y$ ) sets $V_{3} \supset \overline{V_{1}^{Y}}$ and $V_{4} \supset \overline{F_{2}^{Y}}$ such that $V_{3} \cap V_{4}=\varnothing$. Since $V_{3} \cap V_{4}=\varnothing$, we know that $\overline{V_{3}^{Y}} \cap \overline{V_{4}^{Y}} \subseteq \partial V_{3}^{Y} \cap \partial V_{4}^{Y}$. Since $\overline{V_{1}^{Y}} \subseteq V_{3}$ and $V_{3} \cap \partial V_{3}^{Y}=\varnothing$, we know that $\overline{V_{1}^{Y}} \cap \overline{V_{4}^{Y}}=\varnothing$. Recall that $F_{1} \subseteq V_{1}$ and $F_{2} \subseteq V_{4}$. Thus, taking $U_{1}=V_{1}$ and $U_{2}=V_{4}$ we have that $U_{1}$ and $U_{2}$ are disjoint open (in $Y$ ) sets such that $U_{1} \supseteq F_{1}, U_{2} \supseteq F_{2}$ and $\overline{U_{1}^{Y}} \cap \overline{U_{2}^{Y}}=\varnothing$.

Assume now that we have constructed $U_{1}, \ldots, U_{n-1}$ such that $\left\{\overline{U_{i}^{Y}}: 1 \leq i<n\right\}$ is pairwise disjoint and $F_{i} \subset U_{i}$. Apply the argument of $F_{1}$ and $F_{2}$ case above with $\overline{F_{1}^{Y}}$ replaced with $\bigcup_{i<n} \overline{U_{i}^{Y}}$ and $\overline{F_{2}^{Y}}$ replaced with $\overline{F_{n}^{Y}}$ to find a $U_{n}$ such that $\overline{U_{n}^{Y}} \cap \bigcup_{i<n} \overline{U_{i}^{Y}}=\varnothing$. Then, $\mathcal{F}^{\prime}=\left\{U_{1}, \ldots, U_{n}\right\}$ is as desired.

With this, we are now ready to prove the Countable Sum (Compact) Theorem.

Proof of Theorem 7: Let $X=\bigcup_{i<\infty} X_{i}$ where $X$ is metrizable and each $X_{i}$ is a compact $S_{d}(\mathcal{O}, \mathcal{O})$-space. Let $\left(\mathcal{U}_{n}: n<\infty\right)$ be a sequence of open covers of $X$. Partition the natural numbers into infinitely many infinite sets, say $\mathbb{N}=\bigcup_{k<\infty} J_{k}$. Notice that each $\left(\mathcal{U}_{n}: n \in J_{k}\right)$ is a sequence of open covers of $X$.

For each $k$, consider $\left(\mathcal{U}_{n}: n \in J_{k}\right)$ as a sequence of open covers of $X_{k}$. Applying $S_{d}(\mathcal{O}, \mathcal{O})$ of $X_{k}$ to $\left(\mathcal{U}_{n}: n \in J_{k}\right)$ we find an $S_{d}(\mathcal{O}, \mathcal{O})$-refinement $\left(\mathcal{V}_{n}: n \in J_{k}\right)$ of $\left(\mathcal{U}_{n}: n \in J_{k}\right)$. Applying compactness of $X_{k}$ to the open cover $\bigcup_{n \in J_{k}} \mathcal{V}_{n}$ we obtain a finite subcover, say $\mathcal{V}=\left\{V_{1}, \ldots, V_{m}\right\}$. For each $n \in J_{k}$ define

\footnotetext{
${ }^{4}$ Note that for a set $A$ of a topological space $X, \partial A$ denotes the boundary of $A$, where $\partial A=$ $\bar{A} \backslash \operatorname{Int} A=\bar{A} \cap \overline{(X \backslash A)}$.
} 


$$
\mathcal{V}_{n}^{\prime}:= \begin{cases}\varnothing, & \text { if } \mathcal{V} \cap \mathcal{V}_{n}=\varnothing \\ \left\{V_{i}: V_{i} \in \mathcal{V}_{n}, 1 \leq i \leq m\right\}, & \text { otherwise. }\end{cases}
$$

Notice that for each $n \in J_{k}$ we have that $\mathcal{V}_{n}^{\prime}$ is a finite discrete family of sets open in $X_{k}$ refining $\mathcal{V}_{n}$, and so also refining $\mathcal{U}_{n}$.

By recalling that metrizable spaces are normal and that in metrizable spaces, compact subspaces are closed subspaces, for each $n \in J_{k}$, we apply Lemma 6 to each $\mathcal{V}_{n}^{\prime}$ to obtain a finite discrete family $\mathcal{W}_{n}$ of sets open in $X$. For each $V \in \mathcal{V}_{n}^{\prime}$ let $W_{V}$ be the set in $\mathcal{W}_{n}$ such that $V \subseteq W_{V}$. Recall that since $\mathcal{V}_{n}^{\prime}<\mathcal{U}_{n}$, for each $V \in \mathcal{V}_{n}^{\prime}$ there is a $U \in \mathcal{U}_{n}$, say $U_{V}$ such that $V \subseteq U_{V}$. Since $W_{V} \supseteq V$ and $U_{V} \supseteq V$ are both open in $X$, we have that $W_{V} \cap U_{V}$ is open in $X$, and moreover $V \subseteq W_{V} \cap U_{V}$. Doing this for each $V \in \mathcal{V}_{n}^{\prime}$ we find that $\mathcal{W}_{n}^{\prime}=\left\{W_{V} \cap U_{V}: V \in \mathcal{V}_{n}^{\prime}\right\}$ is a discrete family of sets open in $X$ and refining $\mathcal{U}_{n}$. Hence $\left(\mathcal{W}_{n}^{\prime}: n \in J_{k}\right)$ is a sequence of discrete families of sets open in $X$, with each $\mathcal{W}_{n}^{\prime}<\mathcal{U}_{n}$. Since $\bigcup_{n \in J_{k}} \mathcal{V}_{n}^{\prime}$ covers $X_{k}$ and since $\bigcup_{n \in J_{k}} \mathcal{W}_{n}^{\prime} \supseteq \bigcup_{n \in J_{k}} \mathcal{V}_{n}^{\prime}$ we have that $\bigcup_{n \in J_{k}} \mathcal{W}_{n}^{\prime}$ covers $X_{k}$. It is now clear that $\left(\mathcal{W}_{n}^{\prime}: n<\infty\right)$ is a sequence of discrete families of sets open in $X$ such that for each $n, \mathcal{W}_{n}^{\prime}<\mathcal{U}_{n}$ and $\bigcup_{n<\infty} \mathcal{W}_{n}^{\prime}$ is a cover of $X$

\subsection{Zero dimensional spaces}

The only remaining tool needed for our proof that Pol's space is $S_{d}(\mathcal{O}, \mathcal{O})$ is the technique of the proof that

Lemma 7: Every zero-dimensional space is an $S_{d}(\mathcal{O}, \mathcal{O})$-space.

The key to this is the well known result that every open cover of a zero-dimensional space has a refinement which is a disjoint open cover. For instance, see Proposition 
4.2.1 on page 131 of [20]. Then, one observes that every disjoint open cover is a discrete family of open sets. With these in mind, one readily sees that every zero-dimensional space is an $S_{d}(\mathcal{O}, \mathcal{O})$-space.

Proof. Let $X$ be a zero-dimensional space. Let $\left(\mathcal{U}_{n}: n<\infty\right)$ be a sequence of open covers of $X$. For each $n$, by zero-dimensionality of $X$, let $\mathcal{V}_{n}$ be a disjoint open cover of $X$ refining $\mathcal{U}_{n}$. Since $\mathcal{V}_{n}$ is a disjoint open cover of $X$, we see that each $x \in X$ is contained in a single element, say $V$, of $\mathcal{V}_{n}$. Since $x \in V$ and $V$ is an open subset of $X$, we see that $V$ is a neighborhood of $x$. Since $\mathcal{V}_{n}$ is a disjoint family, it is clear that $V$ has trivial intersection with every other element of $\mathcal{V}_{n}$. Hence $\mathcal{V}_{n}$ is a discrete family of open sets. Since each $\mathcal{V}_{n}$ is a cover of $X$, it is clear that $\bigcup_{n<\infty} \mathcal{V}_{n}$ is a cover of $X$. Hence, we see that $\left(\mathcal{V}_{n}: n<\infty\right)$ is an $S_{d}(\mathcal{O}, \mathcal{O})$-refinement of $\left(\mathcal{U}_{n}: n<\infty\right)$, and so $X$ is an $S_{d}(\mathcal{O}, \mathcal{O})$-space.

What we will use from this in the proof of Pol's space is merely that

Fact 6: If $\mathcal{U}$ is an open cover of a zero-dimensional space, say $X$, then there is a $\mathcal{V}$ refining $\mathcal{U}$ such that $\mathcal{V}$ is a discrete family of open sets which is also a cover of $X$.

This was shown in the process of proving Lemma 7. Now, we are ready to prove that Pol's space is an $S_{d}(\mathcal{O}, \mathcal{O})$ space.

\subsection{The proof}

Let $P=L \cup M$ be Pol's space, as described at the start of this chapter. Let $\left(\mathcal{U}_{n}: n<\infty\right)$, a sequence of open covers of $P$, be given. We will write $L=\bigcup_{i<\infty} L_{i}$ where each $L_{i}$ is a compact finite dimensional space, say of dimension $n_{i}$. For each $i$, by the Menger-Nöbeling Theorem, we have that $L_{i}$ is homeomorphic to a subspace, 
say $\mathcal{L}_{i}$ of $\mathbb{R}^{2 n_{i}+1}$. Since $L_{i}$ is compact, so is $\mathcal{L}_{i}$, and hence $\mathcal{L}_{i}$ is a closed subspace of $\mathbb{R}^{2 n_{i}+1}$. By Theorem 3, we know that for each $i<\infty, \mathbb{R}^{2 n_{i}+1}$ is an $S_{d}(\mathcal{O}, \mathcal{O})$-space. Thus, by the Closed Subspace Theorem, we have that $\mathcal{L}_{i}$ is an $S_{d}(\mathcal{O}, \mathcal{O})$-space. Hence, so is $L_{i}$, since $S_{d}(\mathcal{O}, \mathcal{O})$ is preserved by homeomorphisms.

Now that we know that each $L_{i}$ is a compact $S_{d}(\mathcal{O}, \mathcal{O})$-space, we will apply the technique of Theorem 7. Partition the natural numbers such that $\mathbb{N}=\{1\} \cup \bigcup_{i<\infty} J_{i}$ where each $J_{i}$ is an infinite subset of $\mathbb{N} \backslash\{1\}$. For each $i$ consider $\left(\mathcal{U}_{n}: n \in J_{i}\right)$ as a sequence of open covers of $L_{i}$. Apply property $S_{d}(\mathcal{O}, \mathcal{O})$ of $L_{i}$ to $\left(\mathcal{U}_{n}: n \in J_{i}\right)$ to obtain $\left(\mathcal{V}_{n}: n \in J_{i}\right)$, the corresponding $S_{d}(\mathcal{O}, \mathcal{O})$-refinement (in the relative topology on $\left.L_{i}\right)$. Apply compactness of $L_{i}$ to $\bigcup_{n \in J_{i}} \mathcal{V}_{n}$ to obtain a finite subcover say $\mathcal{V}=\left\{V_{1}, \ldots, V_{m}\right\}$. For each $n \in J_{i}$ define

$$
\mathcal{V}_{n}^{\prime}= \begin{cases}\varnothing & \text { if } \mathcal{V} \cap \mathcal{V}_{n}=\varnothing \\ \left\{V_{j}: V_{j} \in \mathcal{V}_{n}, 1 \leq j \leq m\right\} & \text { othewise }\end{cases}
$$

For each $n \in J_{i}$ we have that $\mathcal{V}_{n}^{\prime}$ is a finite discrete family of sets open in $L_{i}$ and refining $\mathcal{U}_{n}$.

Now, for each $n \in J_{i}$, we apply Lemma 6 to each $\mathcal{V}_{n}^{\prime}$ to obtain finite discrete families $\mathcal{W}_{n}$ of sets open in $P$. For each $V \in \mathcal{V}_{n}^{\prime}$ let $W_{V}$ be the set in $\mathcal{W}_{n}$ such that $V \subseteq W_{V}$. Since $\mathcal{V}_{n}^{\prime}<\mathcal{U}_{n}$, for each $V \in \mathcal{V}_{n}^{\prime}$ we pick a $U_{V} \in \mathcal{U}_{n}$ such that $V \subseteq U_{V}$. Hence we see that $\mathcal{W}_{n}^{\prime}=\left\{W_{V} \cap U_{V}: V \in \mathcal{V}_{n}^{\prime}\right\}$ is a discrete family of sets open in $P$ and refining $\mathcal{U}_{n}$. Further, we have that $\bigcup_{n \in J_{i}} \mathcal{W}_{n}^{\prime}$ covers $L_{i}$, and so $\bigcup_{i<\infty}\left(\bigcup_{n \in J_{i}} \mathcal{W}_{n}^{\prime}\right)$ is a cover of $L=\bigcup_{i<\infty} L_{i}$. Additionally, notice that $\mathfrak{W}=\cup\left(\bigcup_{i<\infty}\left(\bigcup_{n \in J_{i}} \mathcal{W}_{n}^{\prime}\right)\right)$ is an open set in $P$, and so $P \backslash \mathfrak{W}$ is closed in $P$, and hence compact, since $P$ is a compact space.

Since $\mathfrak{W}$ covers $L$, we then see that $P \backslash \mathfrak{W}$ is a subset of $M$. Recall that $M$ 
is a totally disconnected space. Since $P \backslash \mathfrak{W}$ is a subset of $M$, which is a totally disconnected space, then by Theorem 29.3 of [29] we see that $P \backslash \mathfrak{W}$ is a totally disconnected space. So $P \backslash \mathfrak{W}$ is a compact, totally disconnected space, and so by Theorem 29.7 of [29] we see that $P \backslash \mathfrak{W}$ is zero-dimensional. Recall that we have yet to use $\mathcal{U}_{1}$. We now do so. Since $\mathcal{U}_{1}$ is a cover of $P$ it is also a cover of $P \backslash \mathfrak{W}$. By Fact 6 we find $\mathcal{V}_{1}$, a discrete family of open sets in $P \backslash \mathfrak{W}$ such that $\mathcal{V}_{1}<\mathcal{U}_{1}$ and is a cover of $P \backslash \mathfrak{W}$. Note that $\mathcal{V}_{1}$ is a pairwise disjoint cover, and so no proper subet of it will be a cover. Hence, by compactness of $P \backslash \mathfrak{W}$ we see that $\mathcal{V}_{1}$ is a finite cover of $P \backslash \mathfrak{W}$. By Lemma 6 applied to $\mathcal{V}_{1}$, we obtain $\mathcal{W}_{1}$, a finite discrete family of sets open in $P$. For each $V \in \mathcal{V}_{1}$ we let $W_{V}$ be the set in $\mathcal{W}_{1}$ such that $V \subseteq W_{V}$. Since $\mathcal{V}_{1}<\mathcal{U}_{1}$, for each $V \in \mathcal{V}_{1}$ we pick a $U_{V} \in \mathcal{U}_{1}$ such that $V \subseteq U$. Hence, we see that $\mathcal{W}_{1}^{\prime}=\left\{W_{V} \cap U_{V}: V \in \mathcal{V}_{1}\right\}$ is a discrete family of sets open in $P$, refining $\mathcal{U}_{1}$ and a cover of $P \backslash \mathfrak{W}$. Thus, we see that $\left(\mathcal{W}_{n}^{\prime}: n<\infty\right)$ is a sequence of discrete families of sets open in $P$ such that for each $n, \mathcal{W}_{n}^{\prime}<\mathcal{U}_{n}$ and $\bigcup_{n<\infty} \mathcal{W}_{n}^{\prime}$ covers $P$. So we may conclude that $P$ is an $S_{d}(\mathcal{O}, \mathcal{O})$-space. 


\section{CHAPTER 3}

\section{OTHER RESULTS}

In the process of examining selective strong screenability, we found additional results not directly connected with showing that Pol's space is an $S_{d}(\mathcal{O}, \mathcal{O})$-space. Among these are free sum theorems for spaces with the Hurewicz property, the Menger property, and selective strong screenability. There are a handful of results about a natural weakening of selective strong screenability for use in topological groups. And, there are some results relating selective strong screenability to other selection principles.

\subsection{Generalizations of earlier results}

In $\S 2.2$, we used the Menger-Nöbeling theorem in concluding that every compact, finite dimensional metric space is an $S_{d}(\mathcal{O}, \mathcal{O})$-space, Theorem 6 . We may generalize this to

Theorem $8(\star)$ : Each finite dimensional metric space with the Hurewicz property is an $S_{d}(\mathcal{O}, \mathcal{O})$-space.

For this, we will need

Lemma 8: The strong Haver property is preserved under subspaces.

Proof. Let $(X, d)$ be a strong Haver space and let $Y$ be a subspace of $X$. Let 
$\left(\varepsilon_{n}: n<\infty\right)$ be a sequence of positive real numbers. Apply the strong Haver property of $X$ to $\left(\varepsilon_{n}: n<\infty\right)$ to obtain a corresponding sequence $\left(\mathcal{V}_{n}: n<\infty\right)$ such that: for each $n, \mathcal{V}_{n}$ is a discrete family of sets open in $X$; for each $n$, for each $V \in \mathcal{V}_{n}$, the $d$-diameter of $V$ is less than $\varepsilon_{n}$; and, $\bigcup_{n<\infty} \mathcal{V}_{n}$ is a cover of $X$. For each $n$, define $\mathcal{U}_{n}=\left\{V \cap Y: V \in \mathcal{V}_{n}\right\}$ and notice that $\left(\mathcal{U}_{n}: n<\infty\right)$ witnesses that $(Y, d)$ has the strong Haver property.

Proof of Theorem 8: Let $X$ be an $n$-dimensional metric space with the Hurewicz property, where $n<\infty$. By the Menger-Nöbeling theorem we know that $X$ is homeomorphic to a subspace, say $X^{\prime}$ of $\mathbb{R}^{2 n+1}$. Since the Hurewicz property is closed under continuous maps, see [17], we see that $X^{\prime}$ has the Hurewicz property. Since $\mathbb{R}^{2 n+1}$ is $S_{d}(\mathcal{O}, \mathcal{O})$ it is straightforward to see that $\mathbb{R}^{2 n+1}$ has the strong Haver property

(see also Theorem 14 below). By Lemma 8 we see that $X^{\prime}$ also has the strong Haver property. Since $X^{\prime}$ has both the strong Haver property and the Hurewicz property, we conclude by Theorem 1 that $X^{\prime}$ is an $S_{d}(\mathcal{O}, \mathcal{O})$-space. Therefore $X$ is an $S_{d}(\mathcal{O}, \mathcal{O})$-space, since $S_{d}(\mathcal{O}, \mathcal{O})$ is preserved under homeomorphisms.

\subsection{Sum theorems}

As seen in $\S 2.2$, we have that the countable union of compact $S_{d}(\mathcal{O}, \mathcal{O})$-spaces is again $S_{d}(\mathcal{O}, \mathcal{O})$. As commented there, we have thus far been unable to extend that result to one without the compact condition. However, we do have that

Theorem 9 (Free Sum Theorem): The free sum of $S_{d}(\mathcal{O}, \mathcal{O})$-spaces is an $S_{d}(\mathcal{O}, \mathcal{O})$ space.

Recall that the free sum of two disjoint spaces, say $X$ and $Y$, denoted $X+Y$ is the set $X \cup Y$ with the topology such that $U \subseteq X+Y$ is open (in $X+Y$ ) if, and only 
if, $U \cap X$ is open in $X$ and $U \cap Y$ is open in $Y$. We denote by $\sum X_{n}$ the set $\bigcup X_{n}$ with the topology such that $U \subseteq \cup X_{n}$ is open (in $\sum X_{n}$ ) if, and only if, $U \cap X_{n}$ is open in $X_{n}$ for each $n$. With the definition of a free sum in mind, we now prove Theorem 9 .

Proof of Theorem 9: For each $n \in \mathbb{N}$ let $X_{n}$ be an $S_{d}(\mathcal{O}, \mathcal{O})$-space. Set $X=\sum X_{n}$. Let $\left(\mathcal{U}_{n}: n<\infty\right)$ be a sequence of open covers of $X$. Partition the natural numbers into infinitely many infinite sets, say $\mathbb{N}=\bigcup_{k<\infty} J_{k}$. For each $k$, apply property $S_{d}(\mathcal{O}, \mathcal{O})$ of $X_{k}$ to $\left(\mathcal{U}_{n}: n \in J_{k}\right)$ to obtain $\left(\mathcal{V}_{n}: n \in J_{k}\right)$ a corresponding $S_{d}(\mathcal{O}, \mathcal{O})$-refinement (in the relative topology on $X_{k}$ ). Since $\left\{X_{n}\right\}_{n \in \mathbb{N}}$ is a pairwise disjoint family of spaces, we have that each set open in $X_{k}$ is open in $X$ and that a discrete family of sets in $X_{k}$ is also a discrete family of sets in $X$. Hence $\bigcup_{n \in J_{k}} \mathcal{V}_{n}$ is an open (in $X$ ) cover of $X_{k}$. Thus, we see that $\left(\mathcal{V}_{n}: n<\infty\right)$ is an $S_{d}(\mathcal{O}, \mathcal{O})$-refinement (in $\left.X\right)$ of $\left(\mathcal{U}_{n}: n<\infty\right)$. So, $X$ is an $S_{d}(\mathcal{O}, \mathcal{O})$-space.

The same basic technique then gives the analogous result for strong Haver spaces and Menger spaces.

Theorem 10: The free sum of strong Haver spaces is a strong Haver space.

Proof. For each $n \in \mathbb{N}$, let $X_{n}$ be a strong Haver space. Set $X=\sum X_{n}$. Let $\left(\varepsilon_{n}: n<\infty\right)$, a sequence of positive reals, be given. Partition the natural numbers into infinitely many infinite sets, say $\mathbb{N}=\bigcup_{k<\infty} J_{k}$. For each $k$, apply the strong Haver property on $X_{k}$ to the sequence of positive reals $\left(\varepsilon_{n}: n \in J_{k}\right)$ to obtain a sequence $\left(\mathcal{V}_{n}: n \in J_{k}\right)$ such that each $\mathcal{V}_{n}$ is a discrete family of sets open in $X_{k}$, each of diameter less than $\varepsilon_{n}$, and $\bigcup_{n \in J_{k}} \mathcal{V}_{n}$ a cover of $X_{k}$. Since $\left\{X_{n}\right\}_{n \in \mathbb{N}}$ is a pairwise disjoint family of spaces, we have that each $\mathcal{V}_{n}$ is a discrete family of open sets in the topology on $X$. Since the metric on $X$ restricted to $X_{k}$ is the same as the metric on $X_{k}$, we have that the diameters agree. Thus, we see that $\left(\mathcal{V}_{n}: n<\infty\right)$ is a sequence of discrete families 
of sets open in $X$ such that for each $n$ and for each $V \in \mathcal{V}_{n}, V$ has diameter less than $\varepsilon_{n}$ and $\bigcup_{n<\infty} \mathcal{V}_{n}$ covers $X$.

Theorem 11: The free sum of Menger spaces is a Menger space.

Recall that a space $X$ is said to be Menger if for each sequence $\left(\mathcal{U}_{n}: n<\infty\right)$ of open covers of $X$ there is a corresponding sequence $\left(\mathcal{V}_{n}: n<\infty\right)$ such that each $\mathcal{V}_{n}$ is a finite subset of $\mathcal{U}_{n}$ and $\bigcup_{n<\infty} \mathcal{V}_{n}$ is a cover of $X$.

Proof. Let $X=\sum X_{n}$ where each $X_{n}$ is Menger. Let $\left(\mathcal{U}_{n}: n<\infty\right)$ be a sequence of open covers of $X$. Partition the natural numbers into infinitely many infinite sets, say $\mathbb{N}=\bigcup_{k<\infty} J_{k}$. For each $k$, apply the Menger property on $X_{k}$ to the sequence $\left(\mathcal{U}_{n}: n \in J_{k}\right)$ to obtain $\left(\mathcal{V}_{n}: n \in J_{k}\right)$ where for each $n \in J_{k}, \mathcal{V}_{n}$ is a finite subset of $\mathcal{U}_{n}$ and $\bigcup_{n \in J_{k}} \mathcal{V}_{n}$ is an open (in the relative topology on $X_{k}$ ) cover of $X_{k}$. Since $\left\{X_{n}\right\}_{n \in \mathbb{N}}$ is a pairwise disjoint family sets open in $X$ we have that each set open in $X_{k}$ is open in $X$. Hence $\bigcup_{n \in J_{k}} \mathcal{V}_{n}$ is an open (in $\left.X\right)$ cover of $X_{k}$. Thus, we see that $\left(\mathcal{V}_{n}: n<\infty\right)$ is such that each $\mathcal{V}_{n}$ is a finite subset of $\mathcal{U}_{n}$ and $\bigcup_{n<\infty} \mathcal{V}_{n}$ is an open cover $X$. So, $X$ is Menger.

Note that the key to the free sum theorems was that both open sets and discrete families are preserved when extended from the topology of the subspace $X_{n}$ to the topology on the space $\sum X_{n}$.

\subsection{More product theorems}

When proving that each finite power of the reals is an $S_{d}(\mathcal{O}, \mathcal{O})$-space in $\S 2.1$, we proved several product theorems about the strong Haver property. Here, we give some product theorems more directly related to when a product of two spaces is an $S_{d}(\mathcal{O}, \mathcal{O})$-space. The first is the corresponding version of Theorem 12 of [4]. 
Theorem 12: If $X$ is a metrizable space with both the strong Haver property and the Hurewicz property, and if $X^{n}$ has the Hurewicz property, then $X^{n}$ is an $S_{d}(\mathcal{O}, \mathcal{O})$ space.

Proof. This is a direct application of Corollary 4 to get that $X^{n}$ has the strong Haver property and then using Theorem 1 to conclude that $X^{n}$ is an $S_{d}(\mathcal{O}, \mathcal{O})$-space.

We highlight the role of compactness in establishing the next theorem.

Theorem 13: If $X$ and $Y$ are $S_{d}(\mathcal{O}, \mathcal{O})$-spaces, at least one of which is compact, then $X \times Y$ is an $S_{d}(\mathcal{O}, \mathcal{O})$-space.

Proof. ${ }^{1}$ Let $X$ and $Y$ be $S_{d}(\mathcal{O}, \mathcal{O})$-spaces with $Y$ a compact space. Consider a sequence of open covers of $X \times Y$, which we will write as $\left(\mathcal{U}_{m, n}: n \in \mathbb{N}, m \in \mathbb{N}\right)$. We may assume that each cover $\mathcal{U}_{m, n}$ is of the form

$$
\mathcal{U}_{m, n}=\left\{A_{m, n}^{\alpha} \times B_{m, n}^{\alpha}: \alpha \in \Gamma_{m, n}\right\}
$$

where $A_{m, n}^{\alpha}$ is open in $X, B_{m, n}^{\alpha}$ is open in $Y$, and $\Gamma_{m, n}$ is an indexing set.

Fix $m \in \mathbb{N}$ and $x \in X$. For each $n \in \mathbb{N}$, by compactness of $Y$, choose a finite indexing set $\Gamma_{m, n}(x) \subseteq \Gamma_{m, n}$ such that

$$
\mathcal{B}_{m, n}(x)=\left\{B_{m, n}^{\alpha}: \alpha \in \Gamma_{m, n}(x)\right\}
$$

is a cover of $Y$ with $x \in A_{m, n}^{\alpha}$ for each $\alpha \in \Gamma_{m, n}(x)$. By $S_{d}(\mathcal{O}, \mathcal{O})$ on $Y$, choose an $S_{d}(\mathcal{O}, \mathcal{O})$-refinement $\left(\mathcal{D}_{m, n}(x): n \in \mathbb{N}\right)$ of $\left(\mathcal{B}_{m, n}(x): n \in \mathbb{N}\right)$. Note that without loss of generality, each $\mathcal{D}_{m, n}(x)$ may be assumed to be finite since each $\mathcal{B}_{m, n}$ is finite.

\footnotetext{
${ }^{1}$ This proof follows that given by Rohm in [26].
} 
By compactness of $Y$ applied to $\bigcup_{n \in \mathbb{N}} \mathcal{D}_{m, n}(x)$ we find finite $R_{m}(x) \subset \mathbb{N}$ such that $\bigcup_{n \in R_{m}(x)} \mathcal{D}_{m, n}(x)$ covers $Y$. Define the following open neighborhood of $x$ :

$$
A_{m}(x)=\bigcap\left\{A_{m, n}^{\alpha}: n \in R_{m}(x), \alpha \in \Gamma_{m, n}(x)\right\} .
$$

For each $m \in \mathbb{N}$ set

$$
\mathcal{A}_{m}:=\left\{A_{m}(x): x \in X\right\}
$$

to obtain a sequence $\left(\mathcal{A}_{m}: m \in \mathbb{N}\right)$ of open covers of $X$.

By $S_{d}(\mathcal{O}, \mathcal{O})$ on $X$ we choose an $S_{d}(\mathcal{O}, \mathcal{O})$-refinement $\left(\mathcal{C}_{m}: m \in \mathbb{N}\right)$ of $\left(\mathcal{A}_{m}: m \in \mathbb{N}\right)$. Since $\mathcal{C}_{m}<\mathcal{A}_{m}$, we choose a function $\phi: \mathcal{C}_{m} \rightarrow X$ such that for each $C \in \mathcal{C}_{m}$ we have that

$$
C \subset A_{m}\left(\phi_{m}(C)\right)
$$

and so for $n \in R_{m}\left(\phi_{m}(C)\right)$ and $\alpha \in \Gamma_{m, n}\left(\phi_{m}(C)\right)$ we have that $C \subset A_{m, n}^{\alpha}$. Now, for each $(m, n) \in \mathbb{N} \times \mathbb{N}$ define

$$
\mathcal{V}_{m, n}=\left\{C \times D:\left(\exists C \in \mathcal{C}_{m}\right)\left(n \in R_{m}\left(\phi_{m}(C)\right)\right) \text { and } D \in \mathcal{D}_{m, n}\left(\phi_{m}(C)\right)\right\}
$$

Notice that $C \in \mathcal{C}_{m}$ means that $C \subset A_{m, n}^{\alpha}$ for each $\alpha \in \Gamma_{m, n}\left(\phi_{m}(C)\right)$. Since $D \in \mathcal{D}_{m, n}\left(\phi_{m}(C)\right)$ we have that $D \subset B_{m, n}^{\alpha}$ for some $\alpha \in \Gamma_{m, n}\left(\phi_{m}(C)\right)$. Thus, for some $\alpha \in \Gamma_{m, n}\left(\phi_{m}(C)\right.$, we have that $C \times D \subset A_{m, n}^{\alpha} \times B_{m, n}^{\alpha} \in \mathcal{U}_{m, n}$. So $\mathcal{V}_{m, n}<\mathcal{U}_{m, n}$.

Now, we claim that since each $\mathcal{C}_{m}$ is a discrete family, as is $\mathcal{D}_{m, n}\left(\phi_{m}(C)\right)$ for a fixed $C \in \mathcal{C}_{m}$, we have that $\mathcal{V}_{m, n}$ is a discrete family. To see this, fix $x \in X$. Let $N_{x}$ be a neighborhood of $x$ witnessing that $\mathcal{C}_{m}$ is a discrete family. If all elements of $\mathcal{C}_{m}$ have empty intersection with $N_{x}$ then $C \times D$, for each $D \in \mathcal{D}_{m, n}\left(\phi_{m}(C)\right)$, has empty intersection with $N_{x} \times Y$. Suppose $C \in \mathcal{C}_{m}$ is such that it is the only such element 
with nontrivial intersection with $N_{x}$. If an element of $\mathcal{V}_{m, n}$ were to have nontrivial intersection with $N_{x} \times N_{y}$, for some open neighborhood $N_{y}$ of $y$, then it must be an element of $\{C\} \times \mathcal{D}_{m, n}\left(\phi_{m}(C)\right)$, which is a discrete family. In either case, we see that $\mathcal{V}_{m, n}$ is a discrete family.

Lastly, we show that $\bigcup\left\{\mathcal{V}_{m, n}: m, n \in \mathbb{N}\right\}$ covers $X \times Y$. Let $(x, y) \in X \times Y$. Since $\bigcup\left\{\mathcal{C}_{m}: m \in \mathbb{N}\right\}$ covers $X$, we pick $m \in \mathbb{N}$ and $C \in \mathcal{C}_{m}$ with $x \in C$. Since $\bigcup\left\{\mathcal{D}_{m, n}\left(\phi_{m}(C)\right): n \in R_{m}\left(\phi_{m}(C)\right)\right\}$ covers $Y$, we pick $n \in R_{m}\left(\phi_{m}(C)\right)$ and $D \in \mathcal{D}_{m, n}\left(\phi_{m}(C)\right)$ such that $y \in D$. Thus, we have that $(x, y) \in C \times D \in \mathcal{V}_{m, n}$.

\subsection{Another relation between strong Haver and $S_{d}(\mathcal{O}, \mathcal{O})$}

As seen in $\S 2.1$, there is a strong connection between the strong Haver property and selective strong screenability. The corresponding connection for the Haver property and $S_{c}(\mathcal{O}, \mathcal{O})$ was commented on by Babinkostova in [4] and was followed up on by E. Pol and R. Pol in [24] where it is stated that

A metrizable space $X$ has property $C$ if and only if for any metric $d$ on $X$ generating the topology, $(X, d)$ has the Haver property.

We found that

Theorem 14: A metrizable space $X$ is $S_{d}(\mathcal{O}, \mathcal{O})$ if, and only if, for any metric $d$ on $X$ generating the topology, $(X, d)$ has the strong Haver property.

The reverse direction is proven using the same method as that given by E. and R. Pol in comment (D) of [24]. It is a straightforward application of definitions to see the forward direction. 
Proof. First, suppose that $X$ is an $S_{d}(\mathcal{O}, \mathcal{O})$-space. Let $d$ be a metric on $X$ generating the topology. Let $\left(\varepsilon_{n}: n<\infty\right)$, a sequence of positive real numbers, be given. For each $n$, let $\mathcal{U}_{n}$ be the cover of $X$ by open balls of $d$-diameter less than $\varepsilon_{n}$. Applying $S_{d}(\mathcal{O}, \mathcal{O})$ of $X$ to $\left(\mathcal{U}_{n}: n<\infty\right)$ gives a sequence $\left(\mathcal{V}_{n}: n<\infty\right)$ such that:

(1) For each $n, \mathcal{V}_{n}$ is a discrete family of sets open in $X$;

(2) For each $n, \mathcal{V}_{n}$ is a refinement of $\mathcal{U}_{n}$;

(3) $\bigcup_{n<\infty} \mathcal{V}_{n}$ is a cover of $X$

Notice (2) gives us that for each $n$ the diameter of elements of $\mathcal{V}_{n}$ is less than $\varepsilon_{n}$. Thus, we see that $(X, d)$ has the strong Haver property.

For the other direction, suppose that for any metric $d$ on $X$ generating the topology, $(X, d)$ has the strong Haver property. Let $(X, d)$ be such a metric space. Suppose that $X$ does not have the $S_{d}(\mathcal{O}, \mathcal{O})$ property. Let $\left(\mathcal{U}_{n}: n<\infty\right)$ be a sequence of open covers of $X$ which witnesses this. Then by Theorem 9.4 of Chapter IX, Section 9 of [12], page 196, we find an equivalent metric $\rho$ such that for each $n$, $\mathcal{W}_{n}=\left\{B_{\rho}\left(x, \frac{1}{n}\right): x \in X\right\}$ refines $\mathcal{U}_{n}$. If $(X, d)$ had the strong Haver property, then we could find a sequence $\left(\mathcal{V}_{n}: n<\infty\right)$ of discrete families such that for each $n$ and for each $V \in \mathcal{V}_{n}$ it happens that $\operatorname{diam} V<\frac{1}{n}$ and $\bigcup_{n<\infty} \mathcal{V}_{n}$ is a cover of $X$. But, then we would have that $\left(\mathcal{V}_{n}: n<\infty\right)$ is an $S_{d}(\mathcal{O}, \mathcal{O})$-refinement of $\left(\mathcal{U}_{n}: n<\infty\right)$, contradicting that $\left(\mathcal{U}_{n}: n<\infty\right)$ witnesses that $(X, d)$ is not $S_{d}(\mathcal{O}, \mathcal{O})$. Thus, $X$ has the $S_{d}(\mathcal{O}, \mathcal{O})$ property.

Recall from Lemma 3 that the cover given by the strong Haver property may be taken to be a large cover, provided the space has no isolated points. Thus, as a corollary to Theorem 14 and Lemma 3 we see that 
Corollary 5: If $(X, d)$ is a selectively strong screenable metric space with no isolated points, then for each sequence $\left(\varepsilon_{n}: n<\infty\right)$ of positive real numbers there is a sequence $\left(\mathcal{U}_{n}: n<\infty\right)$ such that each $\mathcal{U}_{n}$ is a discrete family of open sets, each of diameter less than $\varepsilon_{n}$, and $\bigcup_{n<\infty} \mathcal{U}_{n}$ is a large cover of $X$.

\subsection{Topological Groups}

There is a natural weakening of the property $S_{d}(\mathcal{O}, \mathcal{O})$ for topological groups to the property $S_{d}\left(\mathcal{O}_{n b d}, \mathcal{O}\right)$, read "ess dee oh neighborhood oh". A topological group is a group with a topology such that the group operation and the inverse operation are continuous. The corresponding version for selective screenability was studied by Babinkostova in [6]. For $(G, *)$ a topological group with identity element $e$ and $U$ a neighborhood of $e$ we define $\mathcal{O}(U)=\{x * U: x \in G\}$, which is an open cover of $G$. We denote by $\mathcal{O}_{n b d}$ the collection of all such open covers; explicitly $\mathcal{O}_{n b d}=\{\mathcal{O}(U)$ : $U$ is a neighborhood of $e\}$. With these definitions (which were given in [6]) in mind, we now define the selection principle $S_{d}\left(\mathcal{O}_{n b d}, \mathcal{O}\right)$.

Definition 2: A topological group $G$ is said to have property $S_{d}\left(\mathcal{O}_{n b d}, \mathcal{O}\right)$ if for each sequence $\left(\mathcal{U}_{n}: n<\infty\right)$ of elements of $\mathcal{O}_{n b d}$ there is corresponding sequence $\left(\mathcal{V}_{n}: n<\infty\right)$ such that

(i) For each $n, \mathcal{V}_{n}$ is a discrete family of sets open in $G$.

(ii) For each $n, \mathcal{V}_{n}<\mathcal{U}_{n}$

(iii) $\bigcup_{n<\infty} \mathcal{V}_{n}$ is a cover of $G$.

Many of the results discussed in previous sections also hold for $S_{d}\left(\mathcal{O}_{n b d}, \mathcal{O}\right)$ in topological groups. For instance, the theorem of the previous section becomes 
Theorem 15: Let $(G, *)$ be a metrizable group. The group has property $S_{d}\left(\mathcal{O}_{n b d}, \mathcal{O}\right)$ if and only if the group has the strong Haver property in all equivalent left invariant metrics.

For this, we need the following theorem of Kakutani, as given in [6].

Theorem 16: Let $\left(U_{k}: k<\infty\right)$ be a sequence of subsets of the topological group $(H, *)$ where $\left\{U_{k}: k<\infty\right\}$ is a neighborhood basis of the identity element $e$ and each $U_{k}$ is symmetric (i.e $U_{k}=U_{k}^{-1}$ ), and for each $k$ also $U_{k+1}^{2} \subseteq U_{k}$. Then there is a left invariant metric $d$ on $H$ such that: $d$ is uniformly continuous in the left uniform structure on $H \times H$; if $y^{-1} * x \in U_{k}$ then $d(x, y) \leq\left(\frac{1}{2}\right)^{k-2}$; and, if $d(x, y)<\left(\frac{1}{2}\right)^{k}$ then $y^{-1} * x \in U_{k}$.

We now proceed with the proof of Theorem 15 .

Proof. ${ }^{2}(\Rightarrow)$ Suppose that $(G, *)$ has property $S_{d}\left(\mathcal{O}_{n b d}, \mathcal{O}\right)$. Let $d$ be a left invariant metric on $G$ and let $\left(\varepsilon_{n}: n<\infty\right)$ be a sequence of positive real numbers. For each $n$ choose an open neighborhood $U_{n}$ of the identity element $e$ of $G$ with $\operatorname{diam}_{d}\left(U_{n}\right)<\varepsilon_{n}$ and set $\mathcal{U}_{n}=\mathcal{O}\left(U_{n}\right)$. Notice that $\left(\mathcal{U}_{n}: n<\infty\right)$ is a sequence of elements from $\mathcal{O}_{n b d}(G)$. Thus, applying $S_{d}\left(\mathcal{O}_{n b d}, \mathcal{O}\right)$ we find a sequence $\left(\mathcal{V}_{n}: n<\infty\right)$ of discrete open familes with each $\mathcal{V}_{n}<\mathcal{U}_{n}$ and $\bigcup_{n<\infty} \mathcal{V}_{n}$ a cover of the space.

From the definition of $\mathcal{U}_{n}$ and since $\mathcal{V}_{n}<\mathcal{U}_{n}$ we see that for each $n$ and $V \in \mathcal{V}_{n}$ there is an $x \in G$ with $V \subseteq x * U_{n}$. Thus, since $d$ is a left invariant metric, we have that $\operatorname{diam}_{d}(V) \leq \varepsilon_{n}$. Hence we see that $\left(\mathcal{V}_{n}: n<\infty\right)$ witnesses that $G$ has the strong Haver property.

$(\Leftarrow)$ Assume that $(G, *)$ has the strong Haver property in all equivalent left invariant metrics. Let $\left(\mathcal{U}_{n}: n<\infty\right)$ be an arbitrary sequence of elements from

\footnotetext{
${ }^{2}$ This follows Babinkostova's proof of Theorem 3 of [6].
} 
$\mathcal{O}_{n b d}(G)$. For each $n$ we choose a neighborhood $V_{n}$ of the identity element $e$ of $G$ such that:

(1) For all $n, V_{n} \subset U_{n}$.

(2) For all $n, V_{n} * V_{n} \subset V_{n-1}$.

(3) $\left\{V_{n}: n<\infty\right\}$ is a neighborhood basis of $e$.

By Kakutani's theorem, we choose a left invariant metric $d$ so that for every $n$ :

(4) If $y^{-1} * x \in V_{n}$ then $d(x, y) \leq\left(\frac{1}{2}\right)^{n-2}$.

(5) If $d(x, y)<\left(\frac{1}{2}\right)^{n}$ then $y^{-1} * x \in V_{n}$.

For each $n$, set $\varepsilon_{n}=\left(\frac{1}{2}\right)^{n}$. By the strong Haver property on $(G, d)$ applied to $\left(\varepsilon_{n}: n<\infty\right)$ we find a sequence $\left(\mathcal{V}_{n}: n<\infty\right)$ such that each $\mathcal{V}_{n}$ is a discrete family of open sets, each of diameter less that $\varepsilon_{n}$ and $\bigcup_{n<\infty} \mathcal{V}_{n}$ is a cover of $G$. Notice that for each $n$ and $V \in \mathcal{V}_{n}$ there is an $x_{V} \in G$ with $V \subseteq x_{V} * V_{n} \subseteq x_{V} * U_{n} \in \mathcal{U}_{n}$. Thus we see that $\mathcal{V}_{n}<\mathcal{U}_{n}$. So we conclude that $\left(\mathcal{V}_{n}: n<\infty\right)$ witnesses that $G$ has property $S_{d}\left(\mathcal{O}_{n b d}, \mathcal{O}\right)$

In [6], Babinkostova presents the selection principle Smirnov $-S_{c}(\mathcal{A}, \mathcal{B})$. We consider the strong version of this selection principle. For $S$ an infinite set, and $\mathcal{A}$ and $\mathcal{B}$ collections of families of subsets of $S$, the selection principle Smirnov $-S_{d}(\mathcal{A}, \mathcal{B})$ is defined as: For each sequence $\left(A_{n}: n<\infty\right)$ of elements of the family $\mathcal{A}$ there exists a positive integer $k<\infty$ and a sequence $\left(B_{n}: n \leq k\right)$ where each $B_{n}$ is a discrete family of sets refining $A_{n}, n \leq k$ and $\bigcup_{j \leq k} B_{j}$ is a member of the family $\mathcal{B}$. Similarly, [6] considers the finitary Haver property, which we also modify to the strong version. A metrizable space $X$ is said to be finitary strong Haver with respect to a metric $d$ 
if there is for each sequence $\left(\varepsilon_{n}: n<\infty\right)$ of positive reals a positive integer $k$ and a sequence $\left(\mathcal{V}_{n}: n \leq k\right)$ where each $\mathcal{V}_{n}$ is a discrete family of open sets, each of diameter less than $\varepsilon_{n}$, such that $\bigcup_{n \leq k} \mathcal{V}_{n}$ is a cover of $X$. With these definitions in mind, we find that the following analog of Theorem 15 also holds, and its proof is quite similar to that of Theorem 15.

Theorem 17: Let $(G, *)$ be a metrizable group. The following are equivalent:

(i) The group has property Smirnov $-S_{d}\left(\mathcal{O}_{n b d}, \mathcal{O}\right)$.

(ii) The group has the finitary strong Haver property in all equivalent left invariant metrics.

Proof. To see that (i) $\Rightarrow\left(\right.$ ii), suppose that $(G, *)$ has property Smirnov $-S_{d}\left(\mathcal{O}_{n b d}, \mathcal{O}\right)$. Let $d$ be a left invariant metric of $G$ and let $\left(\varepsilon_{n}: n<\infty\right)$ be a sequence of positive real numbers. For each $n$ choose an open neighborhood $U_{n}$ of the identity element $e$ of $G$ with $\operatorname{diam}_{d}\left(U_{n}\right)<\varepsilon_{n}$ and put $\mathcal{U}_{n}=\mathcal{O}\left(U_{n}\right)$. Notice that $\left(\mathcal{U}_{n}: n<\infty\right)$ is a sequence of elements from $\mathcal{O}_{n b d}(G)$. Thus, applying Smirnov $-S_{d}\left(\mathcal{O}_{n b d}, \mathcal{O}\right)$ we find a positive integer $k$ and a sequence $\left(\mathcal{V}_{n}: n \leq k\right)$ such that

(a) $(\forall n \leq k)\left(\mathcal{V}_{n}\right.$ is a discrete family of open sets $)$,

(b) $(\forall n \leq k)\left(\mathcal{V}_{n}<\mathcal{U}_{n}\right)$, and

(c) $\bigcup_{n \leq k} \mathcal{V}_{n}$ is a cover of $G$.

Now, for each $n \leq k$ and $V \in \mathcal{V}_{n}$ there is an $x \in G$ with $V \subseteq x * U_{n}$. Thus, since $d$ is a left invariant metric, we have that $\operatorname{diam}_{d}(V) \leq \varepsilon_{n}$. Hence, we see that $\left(\mathcal{V}_{n}: n \leq k\right)$ witnesses that $(G, d)$ has the finitary strong Haver property.

To see that (ii) $\Rightarrow(\mathrm{i})$, suppose that $(G, *)$ has the finitary strong Haver property in all equivalent left invariant metrics. For each $n$, let $U_{n}$ be an open neighborhood of 
the identity element $e$ of $G$ and set $\mathcal{U}_{n}=\mathcal{O}\left(U_{n}\right)$. For each $n$, choose a neighborhood $V_{n}$ of $e$ such that:

(1) $(\forall n<\infty)\left(V_{n} \subset U_{n}\right)$,

(2) $(\forall n<\infty)\left(V_{n} * V_{n} \subset V_{n-1}\right)$, and

(3) $\left\{V_{n}: n<\infty\right\}$ is a neighborhood basis of $e$.

By Kakutani's theorem, we choose a left invariant metric $d$ so that for every $n$ :

(4) If $y^{-1} * x \in V_{n}$ then $d(x, y) \leq\left(\frac{1}{2}\right)^{n-2}$, and

(5) If $d(x, y)<\left(\frac{1}{2}\right)^{n}$ then $y^{-1} * x \in V_{n}$.

For each $n$, set $\varepsilon_{n}=\left(\frac{1}{2}\right)^{n}$. By the finitary strong Haver property on $(G, d)$ applied to $\left(\varepsilon_{n}: n<\infty\right)$ we find a positive integer $k$ and a sequence $\left(\mathcal{V}_{n}: n<\infty\right)$ such that:

(I) $(\forall n \leq k)\left(\mathcal{V}_{n}\right.$ is a discrete family of open sets),

(II) $(\forall n \leq k)\left(\forall V \in \mathcal{V}_{n}\right)\left(\operatorname{diam}_{d}(V)<\varepsilon_{n}\right)$, and

(III) $\bigcup_{n \leq k} \mathcal{V}_{n}$ is a cover of $G$.

Notice that for each $n \leq k$ and $V \in \mathcal{V}_{n}$ there is an $x_{V} \in G$ with $V \subseteq x_{V} * V_{n} \subseteq x_{V} * U_{n} \in \mathcal{U}_{n}$. So $\mathcal{V}_{n}<\mathcal{U}_{n}$. Hence, we conclude that $\left(\mathcal{V}_{n}: n<\infty\right)$ witnesses that $G$ has property Smirnov $-S_{d}\left(\mathcal{O}_{n b d}, \mathcal{O}\right)$

The relation between the Hurewicz property and selective strong screenability is again evident in that

Theorem 18: For a topological group $(G, *)$ with the Hurewicz property, $S_{d}\left(\mathcal{O}_{n b d}, \mathcal{O}\right)$ is equivalent to $S_{d}(\mathcal{O}, \mathcal{O})$. 
Proof. ${ }^{3}$ Let $G(, *)$ be a topological group with identity element $e$. That $S_{d}(\mathcal{O}, \mathcal{O})$ implies $S_{d}\left(\mathcal{O}_{n b d}, \mathcal{O}\right)$ is clear. For the other direction, assume that $(G, *)$ has property $S_{d}\left(\mathcal{O}_{n b d}, \mathcal{O}\right)$. Let $\left(\mathcal{U}_{n}: n<\infty\right)$ be a sequence of open covers of $G$. For each $n$ and each $x \in G$ choose a neighborhood $V(x, n)$ of $e$ such that $x * V(x, n)^{4}$ is a subset of some $U \in \mathcal{U}_{n}$. Put $\mathcal{H}_{n}=\{x * V(x, n): x \in G\}$. Apply the Hurewicz property to $\left(\mathcal{H}_{n}: n<\infty\right)$ to obtain for each $n$ a finite $\mathcal{F}_{n} \subset \mathcal{H}_{n}$ such that for each $g \in G$ the set $\left\{n: g \notin \cup \mathcal{F}_{n}\right\}$ is finite. We will write $\mathcal{F}_{n}=\left\{x_{i}^{n} * V\left(x_{i}^{n}, n\right): i \in I_{n}\right\}$ where $I_{n}$ is a finite indexing set.

For each $n$ define $V_{n}=\bigcap_{i \in I_{n}} V\left(x_{i}^{n}, n\right)$, and notice that $V_{n}$ is a neighborhood of $e$. Partition the natural numbers into infinitely many infinite sets, say $\mathbb{N}=\bigcup_{k<\infty} J_{k}$. For each $k$, apply $S_{d}\left(\mathcal{O}_{n b d}, \mathcal{O}\right)$ to the sequence $\left(\mathcal{O}\left(V_{n}\right): n \in J_{k}\right)$ to obtain a sequence $\left(\mathcal{S}_{n}: n \in J_{k}\right)$, where each $\mathcal{S}_{n}$ is a discrete family of open sets with $\mathcal{S}_{n}<\mathcal{O}\left(V_{n}\right)$ and $\bigcup_{n \in J_{k}} \mathcal{S}_{n}$ a cover of $G$. For each $n$ define $\mathcal{V}_{n}=\left\{S \in \mathcal{S}_{n}:\left(\exists U \in \mathcal{U}_{n}\right)(S \subseteq U)\right\}$, and notice that $\mathcal{V}_{n}$ is a discrete family of open sets which refines $\mathcal{U}_{n}$.

Now, we seek to show that $\bigcup_{n<\infty} \mathcal{V}_{n}$ covers $G$. Fix $g \in G$. Since each element of $G$ is in almost every $\cup \mathcal{F}_{n}$, we pick $N_{g} \in \mathbb{N}$ large enough that for $n \geq N_{g}$ it happens that $g \in \cup \mathcal{F}_{n}$. Next, we pick $k_{g} \in \mathbb{N}$ such that $\min \left(J_{k_{g}}\right) \geq N_{g}$. Since $\bigcup_{n \in J_{k_{g}}} \mathcal{S}_{n}$ covers $G$ we pick an $m \in J_{k_{g}}$ and an $S \in \mathcal{S}_{m}$ with $g \in S$. To finish, we will show that $S \in \mathcal{V}_{m}$.

Since $m \geq N_{g}$ we have that $g \in \cup \mathcal{F}_{m}$. So we pick $i \in I_{m}$ with $g \in x_{i}^{m} * V\left(x_{i}^{m}, m\right)$. Since $S \in \mathcal{S}_{m}$ we pick $h_{m} \in G$ so that $S \subseteq h_{m} * V_{m}=h_{m} *\left(\bigcap_{i \in I_{m}} V\left(x_{i}^{m}, m\right)\right) \subseteq h_{m} *$ $V\left(x_{i}^{m}, m\right)$. Thus we see that $g=x_{i}^{m} * z_{g}=h_{m} * t_{g}$ for some $z_{g}, t_{g} \in V\left(x_{i}^{m}, m\right)$. So $h_{m}=x_{i}^{m} * z_{g} * t_{g}^{-1}$. Lastly, consider any $y \in S$ and choose $t_{y} \in V\left(x_{i}^{m}, m\right)$ with $y=$ $h_{m} * t_{y}$. So $y=x_{i}^{m} *\left(z_{g} * t_{g}^{-1} * t_{y} * e\right)$. Since $z_{g}, t_{g}^{-1}, t_{y}, e \in V\left(x_{i}^{m}, m\right)$ we have that $y \in x_{i}^{m} * V\left(x_{i}^{m}, m\right)^{4}$, which is a subset of some $U \in \mathcal{U}_{m}$. Thus $S \in \mathcal{V}_{m}$, as desired.

For a topological space $X$ and a cover $\mathcal{U}$ of $X$, we say that $\mathcal{U}$ is an $\omega$-cover of $X$ if

\footnotetext{
${ }^{3}$ This follows Babinkostova's proof of Theorem 5 of [6].
} 
$X \notin \mathcal{U}$, for each finite subset $F$ of $X$ there is a $U \in \mathcal{U}$ such that $F \subseteq U$. We denote by $\Omega$ the collection of all $\omega$-covers of the space $X$. Note that for a group $(G, *)$ with identity element $e$ and $G$ not compact, we have $\Omega_{n b d}=\{\Omega(U): U$ a neighborhood of $e\}$ where $\Omega(U)=\{F * U: F \subset G$ finite $\}$. So, as a corollary to Theorem 18 we have

Corollary 6: $S_{d}\left(\Omega_{n b d}, \mathcal{O}\right)$ does not imply $S_{d}\left(\mathcal{O}_{n b d}, \mathcal{O}\right)$.

In the analogous proof given in [6], the following facts are given:

Fact 7: $(C, *)$, the unit circle in the complex plane with complex multiplication, is a compact metrizable group embedding the unit interval $[0,1]$ as a subspace.

Fact 8: $\mathbb{R} \times C^{\mathbb{N}}$, where $(C, *)$ is the unit circle in the complex plane with complex multiplication and $\mathbb{R}$ is the real line with addition, is a Hurewicz-bounded group and has property $S_{1}\left(\Omega_{n b d}, \mathcal{O}\right)$.

A topological group $(H, *)$ is said to be Hurewicz-bounded if there is for each sequence $\left(U_{n}: n \in \mathbb{N}\right)$ of open neighborhoods of $e_{H}$ a sequence $\left(F_{n}: n \in \mathbb{N}\right)$ of finite subsets of $H$ such that each element of $H$ belongs to all but finitely many of the sets $F_{n} * U_{n}$. Also, note that $S_{1}\left(\Omega_{n b d}, \mathcal{O}\right)$ means that for each sequence $\left(\mathcal{U}_{n}: n<\infty\right)$ of elements of $\Omega_{n b d}$ there is a sequence $\left(U_{n}: n<\infty\right)$ such that for each $n$ we have $U_{n} \in \mathcal{U}_{n}$ and $\left\{U_{n}: n<\infty\right\} \in \mathcal{O}$.

Fact 9: The topological group $\mathbb{R} \times C^{\mathbb{N}}$ has the Hurewicz property.

Fact 10: $[0,1]^{\mathbb{N}}$ embeds as a closed subspace into $\mathbb{R} \times C^{\mathbb{N}}$.

Fact 11: $[0,1]^{\mathbb{N}}$ does not have property $S_{c}(\mathcal{O}, \mathcal{O})$.

Proof of Corollary 6. ${ }^{4}$ By Fact $8, \mathbb{R} \times C^{\mathbb{N}}$ has property $S_{1}\left(\Omega_{n b d}, \mathcal{O}\right)$. It is clear that $S_{1}\left(\Omega_{n b d}, \mathcal{O}\right)$ implies $S_{d}\left(\Omega_{n b d}, \mathcal{O}\right)$. By Fact $10,[0,1]^{\mathbb{N}}$ embeds as a closed subspace into $\mathbb{R} \times C^{\mathbb{N}}$, call this closed subspace $A$. By Fact 11 , since $S_{d}(\mathcal{O}, \mathcal{O})$ implies $S_{c}(\mathcal{O}, \mathcal{O})$, we

\footnotetext{
${ }^{4}$ The ideas here follow that of Babinkostova's proof of Theorem 6 in [6].
} 
see that $[0,1]^{\mathbb{N}}$ does not have property $S_{d}(\mathcal{O}, \mathcal{O})$. Hence, since $[0,1]^{\mathbb{N}}$ is homeomorphic to $A$, we see that $A$ does not have property $S_{d}(\mathcal{O}, \mathcal{O})$. Since $S_{d}(\mathcal{O}, \mathcal{O})$ is inherited by closed subspaces, and $A$ is a closed subspace of $\mathbb{R} \times C^{\mathbb{N}}$ which is not $S_{d}(\mathcal{O}, \mathcal{O})$, we see that $\mathbb{R} \times C^{\mathbb{N}}$ does not have property $S_{d}(\mathcal{O}, \mathcal{O})$. Fact 9 lets us use Theorem 18 and Fact 8 to see that $\mathbb{R} \times C^{\mathbb{N}}$ does not have property $S_{d}\left(\mathcal{O}_{n b d}, \mathcal{O}\right)$.

In [6], Babinkostova introduced the c-groupable property. The corresponding strong version is called the $d$-groupable property. An open cover $\mathcal{U}$ of a topological space $X$ is said to be $d$-groupable if there is a partition, $\mathcal{U}=\bigcup_{n<\infty} \mathcal{U}_{n}$, where each $\mathcal{U}_{n}$ is a discrete family of sets open in $X$ and each $x \in X$ is in all but finitely many $\bigcup \mathcal{U}_{n}$. We will use $\mathcal{O}^{d g p}$ to denote the collection of $d$-groupable open covers of the space.

$d$-groupability is a weakening of compactness, and so given the nice structure of topological groups we obtain the following analogous version of Theorem 13 .

Theorem 19: Let $(G, *)$ be a group satisfying $S_{d}\left(\mathcal{O}_{n b d}, \mathcal{O}\right)$. If $(H, *)$ is a group with property $S_{d}\left(\mathcal{O}_{n b d}, \mathcal{O}^{d g p}\right)$, then $(G \times H, *)$ also has $S_{d}\left(\mathcal{O}_{n b d}, \mathcal{O}\right)$.

Proof. ${ }^{5}$ For each $n$ let $\mathcal{U}_{n}$ be an element of $\mathcal{O}_{n b d}(G \times H)$. Each $\mathcal{U}_{n}$ is of the form $\mathcal{U}_{n}=\mathcal{O}\left(U_{n}\right)$, where $U_{n}$ is a neigborhood of the identity element $\left(e_{G}, e_{H}\right)$ of $G \times H$. For each $n$, pick $V_{n} \subseteq G$, a neighborhood of $e_{G}$ and pick $W_{n} \subseteq H$, a neighborhood of $e_{H}$ so that $V_{n} \times W_{n} \subseteq U_{n}$. For each $n$, set $\mathcal{W}_{n}=\mathcal{O}\left(V_{n} \times W_{n}\right)$ and notice that $\mathcal{W}_{n}<\mathcal{U}_{n}$.

For each $n$, put $\mathcal{H}_{n}=\mathcal{O}\left(W_{n}\right)$, which is an element of $\mathcal{O}_{n b d}$ on $H$. Applying $S_{d}\left(\mathcal{O}_{n b d}, \mathcal{O}^{d g p}\right)$ of $(H, *)$ to $\left(\mathcal{H}_{n}: n<\infty\right)$ we obtain a sequence $\left(\mathcal{K}_{n}: n<\infty\right)$ such that $\mathcal{K}_{n}<\mathcal{H}_{n}$, for $i \neq j, \mathcal{K}_{i} \cap \mathcal{K}_{j}=\varnothing$, each $\mathcal{K}_{n}$ is a discrete family of open subsets of $H$ such that each $h \in H$ is in almost every $\bigcup \mathcal{K}_{n}$, and $\bigcup_{n<\infty} \mathcal{K}_{n}$ is a cover of $H$.

\footnotetext{
${ }^{5}$ This proof follows that given by Babinkostova of Theorem 7 in [6].
} 
Likewise, for each $n$, set $\mathcal{G}_{n}=\mathcal{O}\left(V_{n}\right)$, which is an element of $\mathcal{O}_{n b d}$ on $G$. Applying $S_{d}\left(\mathcal{O}_{n b d}, \mathcal{O}\right)$ of $(G, *)$ to $\left(\mathcal{G}_{n}: n<\infty\right)$ we obtain a sequence $\left(\mathcal{J}_{n}: n<\infty\right)$ with $\mathcal{J}_{n}<\mathcal{G}_{n}$ and each $\mathcal{J}_{n}$ is a discrete family of open subsets of $G$ such that $\bigcup_{n<\infty} \mathcal{J}_{n}$ covers $G$ and for each $g \in G$ there are infinitely many $n$ such that $x \in \cup \mathcal{J}_{n}$.

For each $n$, define $\mathcal{V}_{n}=\left\{J \times K: J \in \mathcal{J}_{n}\right.$ and $\left.K \in \mathcal{K}_{n}\right\}$. By Lemma 4, we have that $\mathcal{V}_{n}$ is a discrete family of open sets. Since $\mathcal{G}_{n}=\mathcal{O}\left(V_{n}\right)$ and $\mathcal{H}_{n}=\mathcal{O}\left(W_{n}\right)$, and since $\mathcal{J}_{n}<\mathcal{G}_{n}$ and $\mathcal{K}_{n}<\mathcal{H}_{n}$, it is evident that $\mathcal{V}_{n}<\mathcal{W}_{n}$. Since $\mathcal{W}_{n}<\mathcal{U}_{n}$, we have that $\mathcal{V}_{n}<\mathcal{U}_{n}$. It remains to show that $\bigcup_{n<\infty} \mathcal{V}_{n}$ covers $G \times H$.

Let $(g, h) \in G \times H$. Since each element of $H$ is found in all but finitely many $\cup \mathcal{K}_{n}$, we pick $N_{h} \in \mathbb{N}$ large enough that for all $n \geq N_{h}$ we have that $h \in \cup \mathcal{K}_{n}$. Since each element of $G$ is in infinitely many $\cup \mathcal{J}_{n}$, we pick $N_{g} \geq N_{h}$ such that $g \in \cup \mathcal{J}_{N_{g}}$. Now, pick $J \in \mathcal{J}_{N_{g}}$ with $g \in J$ and pick $K \in \mathcal{K}_{N_{g}}$ with $h \in K$. Thus $(g, h) \in J \times K \in \mathcal{V}_{N_{g}}$ and so $\bigcup_{n<\infty} \mathcal{V}_{n}$ covers $G \times H$.

As an analogue to Lemma 7 about zero-dimensional spaces being $S_{d}(\mathcal{O}, \mathcal{O})$, we find that

Lemma 9: If $(H, *)$ is a metrizable zero-dimensional topological group with no isolated points, then $(H, *)$ has property $S_{d}\left(\mathcal{O}_{n b d}, \mathcal{O}^{d g p}\right)$.

The following lemma will be useful in proving this.

Lemma 10: Let $X$ be a zero-dimensional space with no isolated points and let $U$ be a nonempty open subset of $X$. Then there exist nonempty sets $U_{1}$ and $U_{2}$ with empty boundary (i.e. both closed and open, which we will call clopen) such that $U=U_{1} \cup U_{2}$.

Proof. Since $X$ has no isolated points and $U$ is nonempty, we know that $U$ contains at least two distinct points, say $x_{1}$ and $x_{2}$. Let $\delta=d\left(x_{1}, x_{2}\right)$. Notice that $\delta>0$ since $x_{1} \neq x_{2}$. Since $X$ is zero dimensional, $x_{1}$ has arbitrarily small neighborhoods with 
empty boundary. Hence, we pick $U_{1}$ a clopen neighborhood of $x_{1}$ with $\operatorname{diam} U_{1}<\delta$. Since $U_{1}$ is clopen, so is $U_{2}=U \backslash U_{1}$. Since diam $U_{1}<\delta=d\left(x_{1}, x_{2}\right)$ we see that $x_{2} \notin U_{1}$. Thus $x_{2} \in U_{2}$. Thus we have found disjoint nonempty clopen sets, $U_{1}$ and $U_{2}$ whose union is $U$.

Proof of Lemma 9. Let $(H, *)$ be a metrizable zero-dimensional topological group with no isolate points. Since $H$ is zero-dimensional, we have that each open cover of $H$ has a refinement by a disjoint open cover. Note that a disjoint open cover is a discrete family of open sets. Thus, for a given sequence $\left(\mathcal{U}_{n}: n<\infty\right)$ for $\mathcal{O}_{n b d}$ for $H$ we can choose for each $n$ a disjoint open refinement $\mathcal{V}_{n}$ which covers $H$, and by the previous comment each $\mathcal{V}_{n}$ is a discrete family of open sets. We will now recursively construct a sequence $\left(\mathcal{W}_{n}: n<\infty\right)$ witnessing that $\bigcup_{n<\infty} \mathcal{V}_{n}$ is $d$-groupable. Set $\mathcal{W}_{1}=\mathcal{V}_{1}$ and notice that $\mathcal{W}_{1}$ is a discrete family of open sets covering $H$. Set $\mathcal{W}_{2}=\left(\mathcal{V}_{2} \backslash \mathcal{W}_{1}\right) \cup\left(\bigcup_{V \in \mathcal{W}_{1} \cap \mathcal{V}_{2}}\left\{V_{1}, V_{2}\right\}_{V}\right)$ where for each $V \in \mathcal{W}_{1} \cap \mathcal{V}_{2}$ the set $\left\{V_{1}, V_{2}\right\}_{V}$ is given by Lemma 10 . Notice that $\mathcal{W}_{2}$ is also a discrete family of open sets covering $H$. Notice that by doing this we have that $\mathcal{W}_{1} \cap \mathcal{W}_{2}=\varnothing$. Suppose we have constructed $\mathcal{W}_{1}, \mathcal{W}_{2}, \ldots \mathcal{W}_{n}$, discrete families of open sets, such that $\left\{\mathcal{W}_{i}\right\}_{i=1}^{n}$ is a pairwise disjoint family of open covers of $H$. Let $\mathfrak{W}_{n}=\bigcup_{i=1}^{n} \mathcal{W}_{i}$. Set $\mathcal{W}_{n+1}=$ $\left(\mathcal{V}_{n+1} \mid \mathfrak{W}_{n}\right) \cup\left(\bigcup_{V \in \mathfrak{W}_{n} \cap \mathcal{V}_{n+1}}\left\{V_{1}, V_{2}\right\}_{V}\right)$ where for each $V \in \mathfrak{W}_{n} \cap \mathcal{V}_{n+1}$ the set $\left\{V_{1}, V_{2}\right\}_{V}$ is given by Lemma 10 . Notice that $\mathcal{W}_{n+1}$ is a discrete family of open sets covering $H$ and that $\left\{\mathcal{W}_{i}\right\}_{i=1}^{n+1}$ is a pairwise disjoint family of open covers of $H$. As constructed, it is evident that $\bigcup_{n<\infty} \mathcal{W}_{n}$ is $d$-groupable.

From a direct application of Theorem 19 and Lemma 9 we then also have 
Corollary 7: Let $\left(G, *_{1}\right)$ and $\left(H, *_{2}\right)$ be metrizable topological groups such that $\left(G, *_{1}\right)$ has $S_{d}\left(\mathcal{O}_{n b d}, \mathcal{O}\right)$ and $H$ is zero-dimensional with no isolated points. Then $(G \times H, *)$ is a group with property $S_{d}\left(\mathcal{O}_{n b d}, \mathcal{O}\right)$.

Recall that when working with selective strong screenability and the strong Haver property, it was helpful to know that the cover given by the strong Haver property could be taken to be a large cover. ${ }^{6}$ Similarly we find that

Theorem 20: Let $(G, *)$ be a metrizable topological group with no isolated points. Then $S_{d}\left(\mathcal{O}_{n b d}, \mathcal{O}\right)$ is equivalent to $S_{d}\left(\mathcal{O}_{n b d}, \Lambda\right)$.

Proof. ${ }^{7}$ It clear that $S_{d}\left(\mathcal{O}_{n b d}, \Lambda\right)$ implies $S_{d}\left(\mathcal{O}_{n b d}, \mathcal{O}\right)$. For the other direction, let $\left(\mathcal{O}\left(U_{n}\right): n<\infty\right)$ be a sequence in $\mathcal{O}_{n b d}(G)$. Choose a sequence $\left(\varepsilon_{n}: n<\infty\right)$ of positive real numbers converging to zero and such that $\varepsilon_{i}>\varepsilon_{i+1}$ for all $i<\infty$ and $\operatorname{diam}_{d}\left(U_{1} \cap U_{2} \cap \cdots \cap U_{n}\right)>\varepsilon_{n}$ for all $n$. Define $\left(\mathcal{O}\left(V_{n}\right): n<\infty\right)$ such that $\operatorname{diam}_{d}\left(V_{n}\right) \leq \varepsilon_{n}$ for $n<\infty$. Partition the natural numbers into infinitely many infinite sets, say $\mathbb{N}=\bigcup_{k<\infty} J_{k}$. For each $k$, apply $S_{d}\left(\mathcal{O}_{n b d}, \mathcal{O}\right)$ to the sequence $\left(\mathcal{O}\left(V_{n}\right): n \in J_{k}\right)$ to get $\left(\mathcal{O}\left(T_{n}\right): n \in J_{k}\right)$, an $S_{d}\left(\mathcal{O}_{n b d}, \mathcal{O}\right)$-refinement of $\left(\mathcal{O}\left(V_{n}\right): n \in J_{k}\right)$. We seek to show that $\bigcup_{n<\infty} T_{n}$ is a large cover of $G$.

Let $g \in G$ and pick $k_{1} \in J_{1}$ with $g \in \cup T_{k_{1}}$. Next, pick $W_{1} \in T_{k_{1}}$ with $g \in W_{1}$. Since $\left(\varepsilon_{n}: n<\infty\right)$ is a strictly decreasing sequence of positive real numbers which converges to zero, we pick $N_{1}$ such that for $n \geq N_{1}$ we have that $\varepsilon_{n}<\operatorname{diam}_{d}\left(W_{1}\right)$.

Now, pick $J_{2}$ such that $\min J_{2} \geq N_{1}$. Then, choose $k_{2} \in J_{2}$ with $g \in \cup T_{k_{2}}$. Pick $W_{2} \in T_{k_{2}}$ with $g \in W_{2}$. Since $k_{2} \geq N_{1}$, we see that $\varepsilon_{k_{2}}<\operatorname{diam}_{d}\left(W_{1}\right)$. So, by definition of $\mathcal{O}\left(V_{k_{2}}\right)$ we have that $\operatorname{diam}_{d}\left(W_{2}\right) \leq \operatorname{diam}_{d}\left(V_{k_{2}}\right) \leq \varepsilon_{k_{2}}<\operatorname{diam}_{d}\left(W_{1}\right)$. Next, pick $N_{2}$

\footnotetext{
${ }^{6}$ Note that for a topological space we denote the collection of all large open covers of the space by $\Lambda$.

${ }^{7}$ This follows Babinkostova's proof of Theorem 12 of [6].
} 
such that for $n \geq N_{2}$ we have that $\varepsilon_{n}<\operatorname{diam}_{d}\left(W_{2}\right)$, and continue in the same way as we did with $N_{1}$.

Continuing like this we find $W_{1}, W_{2}, W_{3}, \ldots$ infinitely many distinct elements of $\bigcup_{n<\infty} T_{n}$ containing $g$.

\subsection{Relation to other selection principles}

We will follow the notation used in [27]. A topological space is said to have the Rothberger property, denoted $S_{1}(\mathcal{O}, \mathcal{O})$ if for each sequence $\left(\mathcal{U}_{n}: n<\infty\right)$ of open covers, there is a sequence $\left(U_{n}: n<\infty\right)$ where $(\forall n)\left(U_{n} \in \mathcal{U}_{n}\right)$ and $\left\{U_{n}: n<\infty\right\}$ covers $X$. As indicated in the following diagram, the selective strong screenability property is somewhere between the Rothberger property and selective screenability.

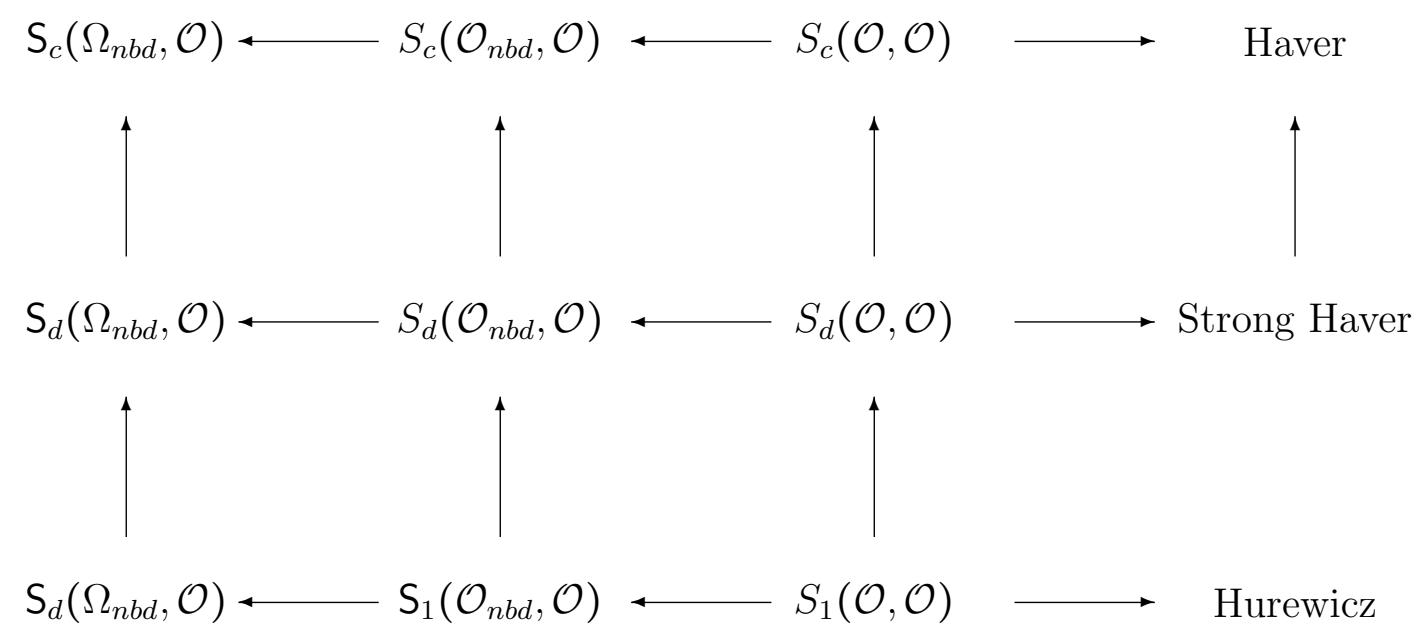

Figure 3.1: Relations between $S_{1}(\mathcal{O}, \mathcal{O}), S_{d}(\mathcal{O}, \mathcal{O})$, and $S_{c}(\mathcal{O}, \mathcal{O})$

Recall that in the previous section we examined the weakening of $S_{d}(\mathcal{O}, \mathcal{O})$ to $S_{d}\left(\mathcal{O}_{n b d}, \mathcal{O}\right)$ for topological groups and saw by Corollary 6 that $S_{d}\left(\Omega_{n b d}, \mathcal{O}\right)$ does not 
imply $S_{d}\left(\mathcal{O}_{n b d}, \mathcal{O}\right)$. Note how this contrasts with the following theorem.

Theorem 21: For a topological space $X$ the following statements are equivalent:

(i) $X$ has property $S_{d}(\mathcal{O}, \mathcal{O})$

(ii) $X$ has property $S_{d}(\Omega, \mathcal{O})$.

Proof. ${ }^{8}$ Since an $\omega$-cover is an open cover, it is clear that $X$ having property $S_{d}(\mathcal{O}, \mathcal{O})$ implies that $X$ has property $S_{d}(\Omega, \mathcal{O})$.

For the other direction, let $\left(\mathcal{U}_{n}: n<\infty\right)$ be a sequence of open covers of $X$. Partition the natural numbers into infinitely many infinite sets, say $\mathbb{N}=\bigcup_{k<\infty} J_{k}$. For each $k<\infty$, for each $i \in J_{k}$ define $\mathcal{W}_{i}$ to be the set whose elements are of the form $\bigcup_{f \in F} U_{f}$, where $F \subset J_{K}$ is finite and $U_{f}$ is an element $\mathcal{U}_{i}$.

For the first case, if there is an $i \in J_{k}$ for which $X \in \mathcal{W}_{i}$, then $X=\bigcup_{f \in F} U_{f}$, where $F \subset J_{K}$ is finite.

For each $f \in F$ set $\mathcal{V}_{f}=\left\{U_{f}\right\}$, and for $j \in \mathbb{N} \backslash F$ pick $U_{j} \in \mathcal{U}_{j}$ arbitrarily and set $\mathcal{V}_{j}=\left\{U_{j}\right\}$. Then the sequence $\left(\mathcal{V}_{n}: n<\infty\right)$ is such that each $\mathcal{V}_{n}$ is a discrete family of open sets refining $\mathcal{U}_{n}$, and $\bigcup_{n<\infty} \mathcal{V}_{n} \supseteq \bigcup_{i \leq \ell} \mathcal{V}_{n_{i}}=\{X\}$, and so is a cover of $X$. Thus, we have shown that, in this case, $X$ has property $S_{d}(\mathcal{O}, \mathcal{O})$.

For the other case, assume that for each $n<\infty, X \notin \mathcal{W}_{n}$. Then each $\mathcal{W}_{n}$ is an $\omega$-cover of $X$. Now, apply $S_{d}(\Omega, \mathcal{O})$ of $X$ to $\left(\mathcal{W}_{n}: n<\infty\right)$ to choose a sequence $\left(\mathcal{T}_{n}: n<\infty\right)$ such that each $\mathcal{T}_{n}$ is a discrete family of open sets refining $\mathcal{W}_{n}$, and $\bigcup_{n<\infty} \mathcal{T}_{n}$ is a cover of $X$. For each $k$ and for each $U \in \mathcal{T}_{k}$ pick $F(U) \in \mathcal{W}_{k}$ such that $U \subseteq F(U)$. Fix for each such $U$ a representation

$$
F(U)=\bigcup_{i \in I_{U}} U_{i}
$$

\footnotetext{
${ }^{8}$ This follows Babinkostova's proof of Theorem 1 of [2].
} 
where $I_{U} \subset J_{k}$ is finite. Then $\mathcal{T}_{k}$ is used as follows to define a refinement for each $\mathcal{U}_{j}$ with $j \in J_{k}$.

For each $i \in J_{k}$, we define $\mathcal{V}_{i}$ in the following way: If $U \in \mathcal{T}_{k}$ has $i \in I_{U}$ we put $S_{i}(U)=U \cap U_{i}$. Then we define $\mathcal{V}_{i}=\left\{S_{i}(U): U \in \mathcal{T}_{k}\right.$ and $\left.i \in I_{U}\right\} \backslash\{\varnothing\}$.

Claim 1: For each $k \in \mathbb{N}$, for each $i \in J_{k}, \mathcal{V}_{i}$ is a discrete family of open sets.

Proof: Fix $k \in \mathbb{N}$. Fix $i \in J_{k}$. Let $\mathcal{U}^{\prime}=\left\{U \in \mathcal{T}_{k}: i \in I_{U}\right\}$. Notice that $\mathcal{U}^{\prime} \subseteq \mathcal{T}_{k}$. Since $\mathcal{T}_{k}$ is a discrete family of open sets, so is $\mathcal{U}^{\prime}$. Notice that $S_{i}\left(U^{\prime}\right)=U^{\prime} \cap U_{i} \subset U^{\prime}$, where $U^{\prime} \in \mathcal{U}^{\prime}$. Further, notice that $\mathcal{V}_{i}=\left\{S_{i}\left(U^{\prime}\right): U^{\prime} \in \mathcal{U}^{\prime}\right\}$. For the sake of contradiction, suppose that $\mathcal{V}_{i}$ is not a discrete family. Then for some $x \in X$ there is a neighborhood of $x$, say $N_{x}$ such that $N_{x}$ has non-trivial intersection with at least two members of $\mathcal{V}_{I}$, say $A$ and $B$. Since $A, B \in \mathcal{V}_{i}$ there exists $U_{A}^{\prime}, U_{B}^{\prime} \in \mathcal{U}^{\prime}$ such that $A=S_{i}\left(U_{A}^{\prime}\right)$ and $B=S_{i}\left(U_{B}^{\prime}\right)$. Notice that $A=S_{i}\left(U_{A}^{\prime}\right)=U_{A}^{\prime} \cap U_{i} \subseteq U_{A}^{\prime}$ and $B=S_{i}\left(U_{B}^{\prime}\right)=U_{B}^{\prime} \cap U_{i} \subseteq U_{B}^{\prime}$. Since $N_{x} \cap A \neq \varnothing$ and $A \subseteq U_{A}^{\prime}$ we have that $N_{x} \cap U_{A}^{\prime} \neq \varnothing$. Similarly $N_{x} \cap U_{B}^{\prime} \neq \varnothing$. Hence, $N_{x}$ has nontrivial intersection with both $U_{A}^{\prime}$ and $U_{B}^{\prime}$, contradicting that $\mathcal{U}^{\prime}$ is a discrete family since $U_{A}^{\prime} \neq U_{B}^{\prime}$. Therefore, we may conclude that for each $k \in \mathbb{N}$, for each $i \in J_{k}, \mathcal{V}_{i}$ is a discrete family of open sets.

Claim 2: $\bigcup_{i<\infty} \mathcal{V}_{i}$ is a cover of $X$.

Proof: Let $x \in X$. Since $\bigcup_{n<\infty} \mathcal{T}_{n}$ is a cover of $X$, there is a $k \in \mathbb{N}$ such that $x \in \cup \mathcal{T}_{k}$. Pick $U \in \mathcal{T}_{k}$ such that $x \in U$. For some $i \in \mathbb{N}$ it happens that $i \in I_{U} \subset J_{k}$ and $x \in U_{i}$. Thus, $S_{i}(U)=U \cap U_{i} \neq \varnothing$ and $x \in S_{i}(U) \in \mathcal{V}_{i} \subset \bigcup_{i<\infty} \mathcal{V}_{i}$. Therefore, $\bigcup_{i<\infty} \mathcal{V}_{i}$ is a cover of $X$. Claim 3: For each $i<\infty, \mathcal{V}_{i}$ refines $\mathcal{U}_{i}$.

Proof: This is clear by noting that each element of $\mathcal{V}_{i}$ is of the form $S_{i}(U)=U \cap U_{i} \subseteq U_{i} \in \mathcal{U}_{i}$.

Thus, Claims 1,2 , and 3 show that $\left(\mathcal{V}_{i}: i<\infty\right)$ witnesses that $X$ has property $S_{d}(\mathcal{O}, \mathcal{O})$ 


\section{CHAPTER 4}

\section{OPEN QUESTIONS}

AND WHERE WE GO FROM HERE

\subsection{The Discrete Extension Question}

Note that the proofs of most of the theorems given above are nearly identical to those given in the literature for the analogous versions for selective screenability. For the product theorems, we were able to do this because of Lemma 4 . This gave us that, just as the product of two disjoint families of sets is still a disjoint family, so is the product of a two discrete family of sets again a discrete family. The main difficulty that was encountered was in trying to show that the countable union of $S_{d}(\mathcal{O}, \mathcal{O})$-spaces is again an $S_{d}(\mathcal{O}, \mathcal{O})$-space. The key to the Countable Sum Theorem for $S_{c}(\mathcal{O}, \mathcal{O})$-spaces is that each disjoint family of sets open in the subspace topology extends nicely to a disjoint family of sets open in the superspace topology. More explicitly, this idea is given by Theorem II.21.XI.2 in [19] as

Theorem 22: Let $Y$ be a nonempty subset of a metric space $X$. Given a family $\left\{U_{\iota}\right\}$ of sets open in $Y$ there exists a family $\left\{V_{\iota}\right\}$ of sets open in $X$ such that $Y \cap V_{\iota}=U_{\iota}$ and for every (finite) system of indices, $\iota_{1}, \ldots, \iota_{n}$, the condition $U_{\iota_{1}} \cap \cdots \cap U_{\iota_{n}}=\varnothing$ implies $V_{\iota 1} \cap \cdots \cap V_{\iota_{n}}=\varnothing$.

The case for families of pairwise disjoint open sets is an application of this theorem 
with $n=2$. If there were an analogous version this theorem for discrete families of open sets, then we would be able to prove the Countable Sum Theorem for $S_{d}(\mathcal{O}, \mathcal{O})$-spaces following the same basic technique as in the case for $S_{c}(\mathcal{O}, \mathcal{O})$-spaces.

Originally, the Countable Sum Theorem for $S_{c}(\mathcal{O}, \mathcal{O})$-spaces was given by Addis and Gresham in [1]. In their paper, Addis and Gresham define a family $\left\{F_{\alpha}: \alpha \in A\right\}$ of subsets of a space $X$ to be separated if for each $\alpha \in A, \bar{F}_{\alpha} \cap\left(\bigcup_{\beta \neq \alpha} F_{\beta}\right)=\varnothing$ and $F_{\alpha} \cap\left(\overline{\bigcup_{\beta \neq \alpha} F_{\beta}}\right)=\varnothing$. It should be noted that a separated family is not necessarily a discrete family. For example in $\mathbb{R}$ with the standard topology, the family $\{(0,1),(1,2)\}$ is separated, but not discrete. However, it is clear that every discrete family is a separated family.

They go on to call a space strongly completely normal if for every separated family $\left\{F_{\alpha}: \alpha \in A\right\}$ in $X$ there exists a disjoint open family $\left\{U_{\alpha}: \alpha \in A\right\}$ such that $F_{\alpha} \subseteq U_{\alpha}$ for each $\alpha \in A$. Addis and Gresham then give the Countable Sum Theorem for $C$-spaces (i.e. $S_{c}(\mathcal{O}, \mathcal{O})$-spaces) as

Let $X$ be a strongly completely normal space such that $X=\bigcup_{n=1}^{\infty} X_{n}$ and each $X_{n}$ is a $C$-space (in the relative topology). Then $X$ is a $C$-space.

After their proof, they go on to note that the assumption of strong complete normality of the space is necessary for their proof. It is unclear whether a condition analogous to strong complete normality is needed for the Countable Sum Theorem for $S_{d}(\mathcal{O}, \mathcal{O})$ spaces.

The following definitions will be useful in discussing the discrete extension question. For families $\mathcal{A}=\left\{A_{\iota}\right\}_{\iota \in I}$ and $\mathcal{B}=\left\{B_{\iota}\right\}_{\iota \in I}$ of a topological space, we say that $\mathcal{A}$ is a precise refinement of $\mathcal{B}$ if $\mathcal{A}<\mathcal{B}$ and for each $\iota \in I, \mathcal{A}_{\iota} \subseteq \mathcal{B}_{\iota}$. For $Y$ a topological space, $X \subseteq Y$ and $\mathcal{F}$ a discrete family of sets open in $X$, we will say that $\mathcal{G}$ is a discrete 
extension of $\mathcal{F}$ in $Y$ if $\mathcal{G}$ is a discrete family of sets open in $Y$ such that $\mathcal{F}$ is a precise refinement of $\mathcal{G}$. Further, we say that $X \subseteq Y$ has the discrete extension property if every discrete family of sets open in $X$ has a discrete extension in $Y$. Note that by Lemma 6 we know that compact subspaces have the discrete extension property. The discrete extension question can then be thought of either as

Question 1: For a topological space $Y$ and $X \subseteq Y$, what conditions on $X$ ensure that $X$ has the discrete extension property?

or as

Question 2: For a topological space $Y$, for what conditions on $Y$ is it true that each subspace of $Y$ has the discrete extension property?

It is clear that if a topological space $X$ is the countable union of $S_{d}(\mathcal{O}, \mathcal{O})$-spaces, each of which has the discrete extension property, then $X$ is an $S_{d}(\mathcal{O}, \mathcal{O})$-space. Is this a necessary condition? Are there well known topological properties which taken together are equivalent to the discrete extension property? It is also clear that if $Y$ is a discrete topological space and $X \subseteq Y$, then $X$ has the discrete extension property. Is there a weaker condition on $Y$ than it being a discrete space that will imply that each subspace of $Y$ has the discrete extension property?

If we proceed by the technique used in the proof of Theorem 7 , then the following lemma shows that a subspace being closed is a necessary condition for it to have the discrete extension property.

Lemma 11: For a metrizable space $Y$, with no isolated points, and a subspace $X$ of $Y$, the following are equivalent:

(a) Every discrete family of sets in $X$ is also a discrete family of sets in $Y$.

(b) $X$ is a closed subspace of $Y$. 
Proof. $(\Leftarrow)$ Let $X$ be a closed subspace of $Y$. Let $\mathcal{F}=\left\{F_{i}: i \in I\right\}$, where $I$ is an indexing set, be a discrete family of sets in $X$. Then $\mathcal{F}^{\prime}=\{\bar{F}: F \in \mathcal{F}\}$ is a pairwise disjoint family of sets in $X$ and for each $J \subseteq I$ the set $\bigcup_{j \in J} \overline{F_{j}}$ is closed in $X$. Since $X$ is a closed subset of $Y$, being closed in $X$ is the same as being closed in $Y$. Thus, it is evident that $\mathcal{F}$ is a discrete family of sets in $Y$.

$\Rightarrow$ Suppose that $X$ is not closed in $Y$. Let $y$ be a limit point of $X$ such that $y \in Y \backslash X$. We will recursively construct a family $\mathcal{F}$ which is discrete in $X$ but not in $Y$. Let $d$ be a metric generating the topology on $Y$. Choose $x_{0} \in X$. Since $y \in Y \backslash X$ we have that $d\left(y, x_{0}\right)=\varepsilon_{0}>0$. Define $F_{0}=B_{d}\left(y, \varepsilon_{0}+1\right) \backslash \overline{B_{d}\left(y, 3 \varepsilon_{0} / 4\right)}$. Since $y$ is a limit point of $X$, we know that $(\exists x \in X)\left(x \in B_{d}\left(y, \varepsilon_{0} / 2\right)\right)$. So, choose $x_{1} \in B_{d}\left(y, \varepsilon_{0} / 2\right) \cap X$ and notice that $d\left(y, x_{1}\right)=\varepsilon_{1}>0$. Define $F_{1}=B_{d}\left(y, \varepsilon_{0} / 2\right) \backslash \overline{B_{d}\left(y, 3 \varepsilon_{1} / 4\right)}$. Notice that $\left\{F_{0}, F_{1}\right\}$ is a discrete family of sets in $X$. Since $y$ is a limit point of $X$, we know that $(\exists x \in X)\left(x \in B_{d}\left(y, \varepsilon_{1} / 2\right)\right)$. So, choose $x_{2} \in B_{d}\left(y, \varepsilon_{1} / 2\right) \cap X$ and notice that $d\left(y, x_{2}\right)=$ $\varepsilon_{2}>0$. Define $F_{1}=B_{d}\left(y, \varepsilon_{1} / 2\right) \backslash \overline{B_{d}\left(y, 3 \varepsilon_{2} / 4\right)}$. Notice that $\left\{F_{0}, F_{1}, F_{2}\right\}$ is a discrete family of sets in $X$. Suppose that we have constructed $\left\{F_{0}, F_{1}, \ldots, F_{n}\right\}$ following this method. Then, since $y$ is a limit point of $X$, we know that $(\exists x \in X)\left(x \in B_{d}\left(y, \varepsilon_{n} / 2\right)\right.$. So, choose $x_{n+1} \in B_{d}\left(y, \varepsilon_{n} / 2\right) \cap X$ and notice that $d\left(y, x_{n+1}\right)=\varepsilon_{n+1}>0$. Define $F_{n+1}=$ $B_{d}\left(y, \varepsilon_{n} / 2\right) \backslash \overline{B_{d}\left(y, 3 \varepsilon_{n+1} / 4\right)}$ and notice that $\left\{F_{0}, F_{1}, \ldots, F_{n+1}\right\}$ is a discrete family of sets in $X$. Set $\mathcal{F}=\left\{F_{n}: n<\infty\right\}$. By the above construction, it is evident that $\mathcal{F}$ is a discrete family of sets in $X$. To see this, let $x \in X$. If $x \in F$ for some $F \in \mathcal{F}$ then take $N_{x}=F$. If $d(x, y) \geq \varepsilon_{0}+1$ then take $N_{x}=B(x, 1)$. If $d(x, y) \in\left[\varepsilon_{n} / 2,3 \varepsilon_{n} / 4\right]$ for some $n \in \mathbb{N}$, then take $N_{x}=B\left(x, \varepsilon_{n+1} / 4\right)$. In each case, $N_{x}$ has nontrivial intersection with at most one element of $\mathcal{F}$. Since $\left(\varepsilon_{n}: n<\infty\right)$, where $\varepsilon_{n}=d\left(y, x_{n}\right)$ with $x_{n}$ as chosen in the above construction, is a strictly decreasing sequence of real numbers which converges to zero, we see that $\mathcal{F}$ fails to be a discrete family of sets in $Y$ since 
every neighborhood of $y$ has nontrivial intersection with infinitely many elements of $\mathcal{F}$.

However, notice that even with this result, the type (open or closed) of discrete family of sets in $X$ is not preserved when viewed as a discrete family of sets in $Y$. To see this, merely let $Y=\mathbb{R}$ and $X=[0,1]$ and take $\mathcal{F}=\{[0,1]\}$ to be the discrete family in $X . \mathcal{F}$ is clearly a discrete family of sets open in $X$, however, since $X$ is closed (and not clopen) in $Y, \mathcal{F}$ is a discrete family of sets closed and not open in $Y$. Even so, it is clear that $\mathcal{F}$ has a discrete extension in $Y$, just take $\mathcal{G}=\{\mathbb{R}\}$. We may preserve the type of discrete family in a discrete extension by first ensuring that the subspace is clopen, as indicated in the following lemma.

Lemma 12: If $Y$ is a topological space and $X \subseteq Y$ is clopen, then every discrete family of sets in $X$ is also a discrete family of sets in $Y$, and the type of discrete family is the same when viewed in $Y$ as in $X$.

The following is a useful example illustrating why the subspace in Lemma 11 needs to be closed.

Example 1: Consider $(0,1]$ as a subspace of $\mathbb{R}$ with the standard topology. Let $\mathcal{F}=\left\{\left(\frac{1}{2^{n+1}}, \frac{1}{2^{n}}\right): n \in \mathbb{N}\right.$ is even $\}$. It is straightforward to show that $\mathcal{F}$ is a discrete family of open sets in $(0,1]$. Let $x \in(0,1]$. If $x \in F$ for some $F \in \mathcal{F}$, then $F$ is a neighborhood of $x$ which has non trivial intersection with at most one element of $\mathcal{F}$. If $x \notin \bigcup_{F \in \mathcal{F}} F$ then either $x=1$ or $x$ lies between two successive elements of $\mathcal{F}$ since $\left(\frac{1}{2^{n}}: n<\infty\right)$ is a strictly decreasing sequence of real numbers. If $x=1$, then take $N_{x}=\left(\frac{1}{2}, 1\right]$. If $x$ lies between two successive elements of $\mathcal{F}$ then $x \in\left(\frac{1}{2^{n_{x}+1}}, \frac{1}{2^{n_{x}}}\right)$ for some odd $n_{x} \in \mathbb{N}$, so take $N_{x}=\left(\frac{1}{2^{n_{x}+1}}, \frac{1}{2^{n_{x}}}\right)$. In either case $N_{x}$ has non trivial 
intersection with at most one element of $\mathcal{F}$ and so $\mathcal{F}$ is a discrete family of open sets in the relative topology on $(0,1]$. However, in $\mathbb{R}$ let $N_{0}$ be any neighborhood of zero. Then diam $N_{0}>0$, so we choose a positive $\varepsilon \leq \operatorname{diam} N_{0}$ such that $B_{d}(0, \varepsilon) \subseteq N_{0}$. In particular, we have that $(0, \varepsilon) \subset N_{0}$. Since $\lim _{n \rightarrow \infty} \frac{1}{2^{n}}=0$, we choose a positive integer $k$ so large that $\frac{1}{2^{k}}<\varepsilon$. Thus for each even $n>k$ it happens that $N_{0} \cap\left(\frac{1}{2^{n+1}}, \frac{1}{2^{n}}\right) \neq \varnothing$. So $N_{0}$ has nontrivial intersection with infinitely many elements of $\mathcal{F}$. Since $N_{0}$ was an arbitrary neighborhood of zero, we see that $\mathcal{F}$ is not a discrete family in $\mathbb{R}$.

The basic idea behind this example is that if the subspace $X$ of $Y$ with metric $d$ does not contain all of its limit points, then for $\ell$ a limit point of $X$ we may construct a discrete family, $\mathcal{F}$ of sets open in $X$ such that inf $\left\{d\left(F, F^{\prime}\right): F, F^{\prime} \in \mathcal{F}\right\}=0$ and $\inf \{d(\ell, F): F \in \mathcal{F}\}=0$. Since $\inf \{d(\ell, F): F \in \mathcal{F}\}=0$, for each neighborhood $N_{\ell}$ of $\ell$, it will happen that there are infinitely many elements of $\mathcal{F}$ which have nontrivial intersection with $N_{\ell}$.

In a footnote of [8], it is remarked that $S_{c}(\mathcal{O}, \mathcal{O})$ and $S_{d}(\mathcal{O}, \mathcal{O})$ are equivalent in normal countably paracompact spaces, and hence in metric spaces. Combining this with Addis and Greshams countable sum theorem as given in [1], it is readily seen that the countable sum theorem for $S_{d}(\mathcal{O}, \mathcal{O})$-spaces holds in spaces which are both countably paracompact and strongly completely normal. Specifically, we have that the countable sum theorem holds for $S_{d}(\mathcal{O}, \mathcal{O})$ metric spaces. Moreover, combined with the Menger-Nöbeling Theorem, we then see that every countable dimensional separable metric space is selectively strongly screenable. 


\subsection{Topological Groups}

The results given in $§ 3.5$ are merely extensions to the strong case of results given by Babinkostova in [6]. There are two additional results from [6] that could be extended by using the basic structure of the proofs given there if we were to first find a positive answer to the question

Question 3: Does Lemma 10 of [6] hold in the strong case?

The strong case of this lemma would be

Conjecture 1: 1 The following statements are equivalent:

1. $X$ has properties $S_{d}(\mathcal{O}, \mathcal{O})$ and Hurewicz.

2. For each sequence $\left(\mathcal{U}_{n}: n<\infty\right)$ of open covers of $X$ there is a sequence $\left(\mathcal{V}_{n}: n<\infty\right)$ such that:

(a) Each $\mathcal{V}_{n}$ is a finite collection of open sets;

(b) Each $\mathcal{V}_{n}$ is a discrete family;

(c) Each $\mathcal{V}_{n}$ refines $\mathcal{U}_{n}$;

(d) There is a sequence $n_{1}<n_{2}<\cdots<n_{k}<\cdots$ of positive integers such that each element of $X$ is in all but finitely many of the sets $\bigcup\left(\bigcup_{n_{k} \leq j<n_{k+1}} \mathcal{V}_{n}\right)$.

The result that would be obtained from proving the first conjecture is that

Conjecture 2: Let $(G, *)$ be a group which has property $S_{d}\left(\mathcal{O}_{n b d}, \mathcal{O}\right)$ as well as the Hurewicz property. Then for any topological group $(H, *)$ satisfying $S_{d}\left(\mathcal{O}_{n b d}, \mathcal{O}\right)$, $(G \times H, *)$ also satisfies $S_{d}\left(\mathcal{O}_{n b d}, \mathcal{O}\right)$.

\footnotetext{
${ }^{1}$ The original version of this lemma for the $S_{c}(\mathcal{O}, \mathcal{O})$ case was proven by Babinkostova and Scheepers in [5].
} 
The first conjecture is merely a nice and neat way of presenting the combination of selective strong screenability and the Hurewicz property for use in the proof of the second conjecture. It should be noted that the first conjecture is likely not actually necessary for the proof of the second conjecture. The key to the proof of the second conjecture appears to be in being able to utilize the "all but finitely many" part of the Hurewicz property. Once we have the second conjecture, one may then use a simple induction argument we then obtain

Conjecture 3: Let $(G, *)$ be a metrizable group which has property $S_{d}\left(\mathcal{O}_{n b d}, \mathcal{O}\right)$ as well as the Hurewicz property. Then all finite powers of $(G, *)$ have property $S_{d}\left(\mathcal{O}_{n b d}, \mathcal{O}\right)$

Obtaining these results would complete examining sections 2 and 3 of [6]. What would then remain to be examined in [6] is section 4 , on games.

As shown in $\S 2.5$, Pol's space is an $S_{d}(\mathcal{O}, \mathcal{O})$-space. It is then natural to ask Question 4: Is the topological group generated by Pol's space an $S_{d}(\mathcal{O}, \mathcal{O})$-space?

\subsection{Relation to other Selection principles}

There are numerous papers addressing the relation between various selection principles, for instance see [27] and [18]. As indicated in Figure 3.1 on page $43, S_{d}(\mathcal{O}, \mathcal{O})$ fits in somewhere between the selection principles $S_{1}(\mathcal{O}, \mathcal{O})$ and $S_{c}(\mathcal{O}, \mathcal{O})$. In Theorem 3 of [8], it is noted that Balogh has an example of a space which is $S_{c}(\mathcal{O}, \mathcal{O})$ but not $S_{d}(\mathcal{O}, \mathcal{O})$. Thus, one would expect that the more general selection principles $S_{c}(\mathcal{A}, \mathcal{B})$ and $S_{d}(\mathcal{A}, \mathcal{B})$ will be quite different from one another. As such, one may which to examine $S_{d}(\mathcal{A}, \mathcal{B})$ in a similar fashion to the treatment that has been done with $S_{c}(\mathcal{A}, \mathcal{B})$ in $[2]$. 


\subsection{Topological Games}

When the author began work on this thesis, there were three main topics to examine. They were: examining selective strong screenablity, primarily in separable metric spaces; examining the natural weakening of $S_{d}(\mathcal{O}, \mathcal{O})$ to $S_{d}\left(\mathcal{O}_{n b d}, \mathcal{O}\right)$ for topological groups; and, examining the topological game related to $S_{d}(\mathcal{O}, \mathcal{O})$ and what relation, if any, it has to dimension theory. The first two topics have been addressed to some extent, but the third has not been explored here. The interest in the topological game related to $S_{d}(\mathcal{O}, \mathcal{O})$ is that for both $S_{1}(\mathcal{O}, \mathcal{O})$ and $S_{c}(\mathcal{O}, \mathcal{O})$ there is much known about their respective topological games (see [21], and [3]) as well as relations between them and dimension theory (see [27], [3], and[7]).

For a topological space $X$ and an ordinal number $\alpha$, the game associated with selective strong screenability is denoted by $G_{d}^{\alpha}(\mathcal{O}, \mathcal{O})$, see [8]. In this game, players ONE and TWO play $\alpha$ innings. In inning $\beta<\alpha$ ONE chooses an open cover $\mathcal{U}_{\beta}$ of $X$ and TWO responds with $\mathcal{V}_{\beta}$ where $\mathcal{V}_{\beta}$ is a discrete family of open sets and $\mathcal{V}_{\beta}<\mathcal{U}_{\beta}$. A play $\left(\left(\mathcal{U}_{\beta}, \mathcal{V}_{\beta}\right): \beta<\alpha\right)$ is won by TWO if $\bigcup_{\beta<\alpha} \mathcal{V}_{\beta}$ covers $X$. Else, ONE wins.

In [8] it was shown that while $S_{c}(\mathcal{O}, \mathcal{O})$ and $S_{d}(\mathcal{O}, \mathcal{O})$ are equivalent in separable metric space, their corresponding games $G_{c}^{\alpha}(\mathcal{O}, \mathcal{O})$ and $G_{d}^{\alpha}(\mathcal{O}, \mathcal{O})$ are quite different in separable metric spaces.

Question 5: What can be said about when player ONE or player TWO have (or do not have) a winning strategy in $G_{d}^{\alpha}(\mathcal{O}, \mathcal{O})$, beyond the results given by Babinkostova and Scheepers in [8]? 


\section{INDEX}

d-groupable, 39

discrete extension, 47-48

discrete extension property, 48

discrete family, 1

finitary strong Haver, 34

free sum, 25

$G_{d}^{\alpha}(\mathcal{O}, \mathcal{O}), 54$

grouping property, 14

Haver property, 5

Hurewicz-bounded, 38

Hurewicz property, 5

isolated point, 10

$\Lambda, 42$

large cover, 10

Menger, 27

normal, 18

$\mathcal{O}^{d g p}, 39$

$\omega$-cover, 37

$\Omega, 38$

$\Omega_{n b d}, 38$

$\mathcal{O}_{n b d}, 32$
Pol's space, 4

precise refinement, 47

property $\mathrm{C}, 1$

$<$, refines, 2

$S_{1}(\mathcal{O}, \mathcal{O})$, Rothberger property, 43

screenable, 1

$S_{c}(\mathcal{O}, \mathcal{O})$, selective screenbility, 2

$S_{d}(\mathcal{O}, \mathcal{O})$, selective strong screenbility, 2

$S_{d}\left(\mathcal{O}_{n b d}, \mathcal{O}\right), 32$

separated, 47

$\sigma$-disjoint grouping property, 9

$\sigma$-discrete grouping property, 9

Smirnov - $S_{d}(\mathcal{A}, \mathcal{B}), 34$

strong Haver property, 5

strongly completely normal, 47

strongly screenable, 1

topological group, 32 


\section{REFERENCES}

[1] D.F. Addis and J.H. Gresham, A class of infinite dimensional spaces Part I: Dimension theory and Alexandroff's problem, Fundamenta Mathematicae 101:3 (1978), $195-205$.

[2] L. Babinkostova, Selective versions of screenability, Filomat 15 (2001), 127 134.

[3] L. Babinkostova, Selective screenability game and covering dimension, Topology Proceedings 29:1 (2005), $13-17$.

[4] L. Babinkostova, When does the Haver property imply selective screenability?, Topology and its Applications 154 (2007), 1971 - 1979.

[5] L. Babinkostova and M. Scheepers, Products and selection principles, Topology Proceedings 31:2 (2007), $431-443$.

[6] L. Babinkostova, Selective screenability in topological groups, Topology and its Applications 186:1 (2008), 2 - 9.

[7] L. Babinkostova, Topological games and covering dimension, Topology Proceedings 38 (2011), 99-120.

[8] L. Babinkostova and M. Scheepers, Selective strong screenability and a game, Topology Proceedings 47 (2016), 297 - 311 (e-published November 1, 2015).

[9] R.H. Bing, Metrization of Topological Spaces, Canadian Journal of Mathematics 3 (1951), 175 - 186.

[10] P. Borst, Classification of weakly infinite dimensional space Part I: A transfinite extension of covering dimension, Fundamenta Mathematicae 130 (1988), 1 -25 .

[11] P. Borst, Some remarks concerning C-spaces, Mathematica Japonica 154 (2007), $665-674$.

[12] J. Dugundji, Topology, Allyn and Bacon, 1966. 
[13] V.V. Fedorchuk, Weakly infinite-dimensional spaces, Russian Mathematical Surveys 42:2 (2007), $1-52$.

[14] V.V. Fedorchuk, Questions on weakly infinite-dimensionl spaces, in: E.M. Pearl (Ed.), Open Problems in Topology II, Elsevier, 2007, 637 - 645.

[15] Y. Hattori and K. Yamada, Closed pre-images of C-spaces, Mathematica Japonica 34:4 (1989), $555-561$.

[16] W.E. Haver, A covering property for metric spaces, Lecture Notes in Mathematics (Springer-Verlag) 275 (1974), 108 - 113.

[17] W. Hurewicz, Über Folgen stetiger Funktionen, Fundamenta Mathematicae 9 (1927), 193 - 204.

[18] W. Just, A.W. Miller, M. Scheepers and P.J. Szeptycki, The combinatorics of open covers (II), Topology and its Applications 73 (1996), 241 - 266.

[19] C. Kuratowski, Topology, vol. 1, Academic Press, 1966.

[20] J. van Mill, Infinite-Dimensional Topology: Prerequisites and Introduction, North-Holland, 1989.

[21] J. Pawlikowski, Undetermined sets of Point-Open Games, Fundamenta Mathematicae 144 (1994), 279-285.

[22] E. Pol, A weakly infinite dimensional space whose product with the irrationals is strongly infinite dimensional, Proceedings of the American Mathematical Society 98:2 (1986), $349-352$.

[23] E. Pol. Spaces whose $n$-th power is weakly infinite dimensional, but whose $n+1$-th power is not, Proceedings of the American Mathematical Society 17:3 (1993), 871 - 876.

[24] E. Pol and R. Pol, A metric space with the Haver property whose square fails this property, Proceedings of the American Mathematical Society 137 (2009) $745-750$.

[25] R. Pol, A weakly infinite-dimensional compactum which is not countabledimensional, Proceedings of the American Mathematical Society 82 (1981), $634-636$.

[26] D.M. Rohm, Products of infinite dimensional spaces, Proceedings of the American Mathematical Society 108:4 (1990), 1019 - 1023. 
[27] M. Scheepers, Combinatorics of open covers I: Ramsey theory, Topology and its Applications 69:1 (1996), 31 - 62.

[28] M. Scheepers, Finite powers of strong measure zero sets, The Journal of Symbolic Logic 64:3 (1999), 1295 - 1306.

[29] S. Willard, General Topology, Addison-Wesley Publishing Company, 1968. 